\title{
Large-time Behavior of Solutions of the Discrete Boltzmann Equation
}

\author{
Shuichi Kawashima ${ }^{\star}$ \\ Department of Mathematics, Nara Women's University, Nara 630, Japan
}

\begin{abstract}
Large-time behavior of solutions of the one-dimensional discrete Boltzmann equation is studied. Under suitable assumptions it is proved that as time tends to infinity, the solution approaches a function which is constructed explicitly in terms of the self-similar solutions of the Burgers equation and the linear heat equation.
\end{abstract}

\section{Introduction}

The one-dimensional discrete Boltzmann equations is written in the form (see Appendix)

$$
\frac{\partial F_{i}}{\partial t}+v_{i} \frac{\partial F_{i}}{\partial x}=\frac{1}{\alpha_{i j, k, l=1}} \sum^{m}\left(A_{k l}^{i j} F_{k} F_{l}-A_{i j}^{k l} F_{i} F_{j}\right), \quad i=1, \ldots, m .
$$

Here $F_{i}=F_{i}(t, x) \geqq 0$ denotes the mass density of gas particles with the velocity $v_{i}$ (real constant) at time $t \geqq 0$ and position $x \in \mathbb{R}$. The coefficients $\alpha_{i}$ are positive constants. Also, $A_{k l}^{i j}$ are nonnegative constants satisfying

$$
A_{l k}^{i j}=A_{k l}^{i j}=A_{k l}^{j i}, \quad A_{k l}^{i j}=A_{i j}^{k l}
$$

for any $i, j, k, l=1, \ldots, m$. In order to exclude the trivial case, we may assume that

$$
A_{k l}^{i j} \neq 0 \text { for some } i, j, k, l=1, \ldots, m \text {. }
$$

We rewrite (1.1) in the vector form. Put $F={ }^{t}\left(F_{1}, \ldots, F_{m}\right) V=\operatorname{diag}\left(v_{1}, \ldots, v_{m}\right)$ and $Q(F, G)={ }^{t}\left(Q_{1}(F, G), \ldots, Q_{m}(F, G)\right)$, where each $Q_{i}(F, G)$ is defined by

$$
Q_{i}(F, G)=\frac{1}{2 \alpha_{i}} \sum_{j, k, l}\left\{A_{k l}^{i j}\left(F_{k} G_{l}+F_{l} G_{k}\right)-A_{i j}^{k l}\left(F_{i} G_{j}+F_{j} G_{i}\right)\right\},
$$

\footnotetext{
^Present address: Department of Applied Science, Faculty of Engineering, Kyushu University 36, Fukuoka 812, Japan
} 
so that $Q_{i}(F, F)$ is just the right-hand side of (1.1). We then arrive at

$$
F_{t}+V F_{x}=Q(F, F)
$$

We consider the initial value problem for (1.5) with the initial data

$$
F(0, x)=F_{0}(x)
$$

It is obvious that $F=0$ is a solution of (1.5). The existence of global solutions of (1.5), (1.6) near the zero solution was proved by Tartar [18] and Cabannes [4] when the initial data $F_{0}(x)$ are nonnegative and small. Asymptotic descriptions for $t \rightarrow \infty$ of the solutions were also given in [18]. (For the generalization of these results to the case of several dimensions, see [9] and the references therein.) It is also known that similar results hold for some concrete models, such as the onedimensional Broadwell model, without smallness conditions on the initial data. See [1] and the references therein.

Let $M$ be a constant vector with positive components and satisfy $Q(M, M)=0$. Such a vector is an equilibrium of (1.5) and is called an absolute Maxwellian. The problem concerning the global existence of solutions of (1.5), (1.6) near absolute Maxwellians was considered in $[13,17]$ under a suitable condition, i.e., Condition 1 in Sect. 3. It was proved that if the initial data $F_{0}(x)$ are near an absolute Maxwellian $M$, then the problem (1.5), (1.6) has a unique global solution $F(t, x)$ which converges to $M$ at the rate $t^{-1 / 4}$ as $t \rightarrow \infty$. See Theorem 3.1. For more details, see [13]. (This result is valid also for the case of several dimensions. In that case, the convergence rate is $t^{-n / 4}$, where $n$ is the spatial dimension. See $[13,17]$.)

The aim of this paper is to give an asymptotic form for $t \rightarrow \infty$ of the solution $F(t, x)$ of $(1.5),(1.6)$ obtained in $[13,17]$. We shall show that $F(t, x)$ approaches a function $\bar{F}(t, x)$ at the rate $t^{-1 / 2+\alpha}, \alpha>0$, as $t \rightarrow \infty$. Here $\bar{F}(t, x)$ is defined by a linear combination of its moments $\bar{w}_{j}(t, x), j=1, \ldots, d$, and $\bar{w}(t, x)={ }^{t}\left(\bar{w}_{1}, \ldots, \bar{w}_{d}\right)$ $(t, x)$ is given by the superposition of the nonlinear and linear diffusion waves constructed by the self-similar solutions of the Burgers equation and the linear heat equation. See Theorem 8.2. To prove this, we require that the Eucler equation (4.8) obtained from (1.5) as the first approximation of the Chapman-Enskog expansion is strictly hyperbolic and each characteristic field is either genuinely nonlinear or linearly degenerate, Condition 2 in Sect. 4. A similar asymptotic behavior of solutions was proved in [14] for a general class of hyperbolic-parabolic systems of conservation equations including the equations of viscous (or inviscid) heat conductive fluids.

We shall explain our approach. After some preparations in Sects. 2-5, we show in Sect. 6 that the solution $F(t, x)$ of $(1.5),(1.6)$ approaches a function $G(t, x)$ at the rate $t^{-3 / 4+\alpha}, \alpha>0$, as $t \rightarrow \infty$ (Theorem 6.2). $G(t, x)$ is a linear combination of its moments $z_{j}(t, x), j=1, \ldots, d$, and $z(t, x)={ }^{t}\left(z_{1}, \ldots, z_{d}\right)(t, x)$ is a unique global solution of the semilinear uniformly parabolic system (6.14), which is determined on the basis of the spectral representation of solutions to the linearized equation of (1.5) around the absolute Maxwellian $M$.

In Sect. 7 we define $G^{\prime}(t, x)$ by a linear combination of its moments $z_{j}^{\prime}(t, x)$ $j=1, \ldots, d$, where $z^{\prime}(t, x)={ }^{t}\left(z_{1}^{\prime}, \ldots, z_{d}^{\prime}\right)(t, x)$ is a unique global solution of the semilinear uniformly parabolic system (7.2) with the same viscosity matrix as in (6.14). The hyperbolic part of (7.2) is exactly the same as the Euler equation (4.8) 
obtained from (1.5). It is then proved that the difference $G^{\prime}(t, x)-G(t, x)$ tends to zero at the rate $t^{-3 / 4+\alpha}, \alpha>0$, as $t \rightarrow \infty$ (Proposition 7.3). This is based on the fact that the difference of the nonlinear terms of these two systems is of the third order (Lemma 7.2).

An asymptotic solution $\bar{w}(t, x)={ }^{t}\left(\bar{w}_{1}, \ldots, \bar{w}_{d}\right)(t, x)$ for $t \rightarrow \infty$ of the system (7.2) was constructed in [14] by employing the technique of Liu [16]. It was given by the superposition of the nonlinear and linear diffusion waves constructed by the self-similar solutions of the Burgers equation and the linear heat equation. We then define $\bar{F}(t, x)$ by a linear combination of $\bar{w}_{j}(t, x), j=1, \ldots, d$, such that $\bar{w}_{j}(t, x)$ become the moments of $\bar{F}(t, x)$. Since the difference $z^{\prime}(t, x)-\bar{w}(t, x)$ tends to zero at the rate $t^{-1 / 2+\alpha}, \alpha>0$, as $t \rightarrow \infty$, we see that $G^{\prime}(t, x)$ approaches $\bar{F}(t, x)$ at the same rate as $t \rightarrow \infty$. Consequently, we know that the solution $F(t, x)$ of (1.5), (1.6) approaches $\bar{F}(t, x)$ at the rate $t^{-1 / 2+\alpha}, \alpha>0$, as $t \rightarrow \infty$, that is, $\bar{F}(t, x)$ is an asymptotic solution for $t \rightarrow \infty$ of (1.5) (Theorem 8.2).

The final section contains an application to the one-dimensional Broadwell model. In the Appendix we give a recipe for reducing the discrete Boltzmann equation in several dimensions into the one-dimensional equation.

\section{Notations}

For $p \in[1, \infty], L^{p}$ denotes the usual Lebesgue space on $\mathbb{R}$ with the norm $\|\cdot\|_{L^{p}}$. When $p=2$, we use the abbreviation $\|\cdot\|=\|\cdot\|_{L^{2}}$. For $\beta \in \mathbb{R}, L_{\beta}^{p}$ denotes the space of functions $u=u(x)$ such that $(1+|x|)^{\beta} u \in L^{p}$, with the norm $\|\cdot\|_{L_{\beta}^{p}}$. Let $s \geqq 0$ be an integer. $H^{s}$ denotes the space of functions $u=u(x)$ such that the derivatives $\partial_{x}^{l} u, 0 \leqq l \leqq s$, are $L^{2}$-functions on $\mathbb{R}$, with the norm $\|\cdot\|_{s}$. Note that $H^{0}=L^{2}$ and $\|\cdot\|_{0}=\|\cdot\|$. We denote by $\mathscr{B}^{s}$ the space of functions $u=u(x)$ such that $\partial_{x}^{l} u, 0 \leqq l \leqq s$, are bounded and continuous on $\mathbb{R}$. Let $I$ be an interval in $[0, \infty)$, and let $k, s \geqq 0$ be integers. $C^{k}\left(I ; H^{s}\right)$ denotes the space of $k$-times continuously differentiable functions on $I$ with values in $H^{s}$. $L^{2}\left(I ; H^{s}\right)$ denotes the space of $L^{2}$-functions on $I$ with values in $H^{s}$.

\section{Preliminaries}

We introduce some basic concepts concerning the discrete Boltzmann equation (1.1) or (1.5). A vector $\psi={ }^{t}\left(\psi_{1}, \ldots, \psi_{m}\right) \in \mathbb{R}^{m}$ is called a summational invariant if

$$
A_{k l}^{i j}\left(\psi_{i} / \alpha_{i}+\psi_{j} / \alpha_{j}-\psi_{k} / \alpha_{k}-\psi_{l} / \alpha_{l}\right)=0
$$

for any $i, j, k, l=1, \ldots, m$. It is known that the following three conditions are equivalent $([8,3])$.

$$
\begin{gathered}
\psi \text { is a summational invariant, } \\
\langle\psi, Q(F, G)\rangle=0 \text { for any } F, G \in \mathbb{R}^{m}, \\
\langle\psi, Q(F, F)\rangle=0 \text { for any } F \in \mathbb{R}^{m},
\end{gathered}
$$

where $\langle$,$\rangle denotes the standard inner product in \mathbb{R}^{m}$. We denote by $\mathscr{M}$ the set of all summational invariants. $\mathscr{M}$ is a subspace of $\mathbb{R}^{m}$ such that $1 \leqq \operatorname{dim} \mathscr{M} \leqq m-1$ 
because ${ }^{t}\left(\alpha_{1}, \ldots, \alpha_{m}\right) \in \mathscr{M}$ and $\mathscr{M} \neq \mathbb{R}^{m}$ by (1.3). Put $d=\operatorname{dim} \mathscr{M}$. Let $\psi^{(J)}, j=1, \ldots, d$, and $\phi^{(k)}, k=d+1, \ldots, m$, be constant vectors such that

$$
\left\{\psi^{(1)}, \ldots, \psi^{(d)}\right\} \text { is a basis of } \mathscr{M} \text {, and }\left\{\phi^{(d+1)}, \ldots, \phi^{(m)}\right\} \text { is a basis of } \mathscr{M}^{\perp} \text {, }
$$

where $\mathscr{M}^{\perp}$ denotes the orthogonal complement of $\mathscr{M}$ in $\mathbb{R}^{m}$. For $F \in \mathbb{R}^{m}$, we define

$$
w={ }^{t}\left(w_{1}, \ldots, w_{d}\right), \quad w_{j}=\left\langle F, \psi^{(j)}\right\rangle, \quad j=1, \ldots, d .
$$

Each $w_{j}$ is called the $j$-th moment of $F$.

Let $F={ }^{t}\left(F_{1}, \ldots, F_{m}\right) \in \mathbb{R}^{m}$ be a vector with positive components. Then $F$ is called a Maxwellian if

$$
A_{k l}^{i j}\left(F_{i} F_{j}-F_{k} F_{l}\right)=0
$$

for any $i, j, k, l=1, \ldots, m$. A constant Maxwellian is called an absolute Maxwellian. It is known that the following three conditions are equivalent $([8,3])$.

$$
\begin{gathered}
F \quad \text { is a Maxwellian, } \\
{ }^{t}\left(\alpha_{1} \log F_{1}, \ldots, \alpha_{m} \log F_{m}\right) \in \mathscr{M}, \\
Q(F, F)=0 .
\end{gathered}
$$

Here the components $F_{i}, i=1, \ldots, m$, are assumed to be positive. The following fact will be used in Sect. 4.

Lemma 2.1 ([8,3]) A Maxwellian $F={ }^{t}\left(F_{1}, \ldots, F_{m}\right)$ with positive components is completely determined by its moments $w={ }^{t}\left(w_{1}, \ldots, w_{d}\right)$.

In fact, from $(2.6)_{1,2}$, there are coefficients $u={ }^{t}\left(u_{1}, \ldots, u_{d}\right) \in \mathbb{R}^{d}$ such that $\alpha_{i} \log F_{i}=\sum_{j=1}^{d} u_{j} \psi_{i}^{(j)}, i=1, \ldots, m$, where $\psi_{i}^{(j)}$ is the $i$-th component of $\psi^{(j)}$. Therefore,

$$
\begin{gathered}
F=\bar{F}(u)={ }^{t}\left(\bar{F}_{1}(u), \ldots, \bar{F}_{m}(u)\right), \\
\bar{F}_{i}(u)=\exp \left(\frac{1}{\alpha_{i}} \sum_{j=1}^{d} u_{j} \psi_{i}^{(j)}\right), \quad i=1, \ldots, m .
\end{gathered}
$$

The moments of $F$ are then expressed in terms of $u$ as follows.

$$
\begin{gathered}
w=w(u)={ }^{t}\left(w_{1}(u), \ldots, w_{d}(u)\right), \\
w_{j}(u)=\left\langle\bar{F}(u), \psi^{(j)}\right\rangle=\sum_{i=1}^{m} \bar{F}_{i}(u) \psi_{i}^{(j)}, \quad j=1, \ldots, d .
\end{gathered}
$$

We denote by $D_{u} w(u)$ the Jacobian of the mapping $w=w(u)$. A simple calculation shows that

$$
D_{u} w(u)=\left(\left\langle\Lambda_{\bar{F}(u)} \psi^{(j)}, \psi^{(k)}\right\rangle\right)_{1 \leqq 1, k \leqq d}
$$

where

$$
\Lambda_{F}=\operatorname{diag}\left(F_{1} / \alpha_{1}, \ldots, F_{m} / \alpha_{m}\right), \quad F={ }^{t}\left(F_{1}, \ldots, F_{m}\right) \in \mathbb{R}^{m}
$$

The matrix with components $\left\langle\Lambda_{F} \psi^{(j)}, \psi^{(k)}\right\rangle, j, k=1, \ldots, d$, is real symmetric and positive definite, provided that all the components of $F$ are positive. Therefore 
$D_{u} w(u)$ is non-singular and hence the inverse mapping $u=u(w)$ exists in each neighborhood. Thus we have the expression $F=\bar{F}(u(w))$ by (2.7). This completes the proof of Lemma 2.1.

Let $M \in \mathbb{R}^{m}$ be a vector with positive components. Put

$$
\begin{aligned}
& L_{M} f=-2 \Lambda_{M}^{-1 / 2} Q\left(M, \Lambda_{M}^{1 / 2} f\right), \quad f \in \mathbb{R}^{m}, \\
& \Gamma_{M}(f, g)=\Lambda_{M}^{-1 / 2} Q\left(\Lambda_{M}^{1 / 2} f, \Lambda_{M}^{1 / 2} g\right), \quad f, g \in \mathbb{R}^{m},
\end{aligned}
$$

where $\Lambda_{M}$ is given by (2.10) with $F=M$.

Lemma 2.2. ([13]) If $M$ is a Maxwellian with positive components, then the following is true. (i) $L_{M}$ is real symmetric and nonnegative definite. Its null space $N\left(L_{M}\right)$ is equal to $\Lambda_{M}^{1 / 2} \mathscr{M}$. (ii) $\Gamma_{M}$ is bi-linear and satisfies $\Gamma_{M}(f, g) \in N\left(L_{M}\right)^{\perp}$ for any $f, g \in \mathbb{R}^{m}$.

When $M$ is a Maxwellian with positive components, we have $N\left(L_{M}\right)=\Lambda_{M}^{1 / 2} \mathscr{M}$ and $N\left(L_{M}\right)^{\perp}=\Lambda_{M}^{-1 / 2} \mathscr{M}^{\perp}$. Therefore,

$$
\begin{gathered}
\left\{\Lambda_{M}^{1 / 2} \psi^{(1)}, \ldots, \Lambda_{M}^{1 / 2} \psi^{(d)}\right\} \quad \text { is a basis of } N\left(L_{M}\right), \\
\left\{\Lambda_{M}^{-1 / 2} \phi^{(d+1)}, \ldots, \Lambda_{M}^{-1 / 2} \phi^{(m)}\right\} \quad \text { is a basis of } N\left(L_{M}\right)^{\perp} .
\end{gathered}
$$

We choose vectors $\phi^{(j)}, j=1, \ldots, d$, (depending on $M$ ) as follows.

$$
\begin{gathered}
\left\{\phi^{(1)}, \ldots, \phi^{(d)}\right\} \quad \text { is a basis of } \Lambda_{M} \mathscr{M}, \\
\left\langle\phi^{(j)}, \psi^{(k)}\right\rangle=\delta_{j k}, \quad j, k=1, \ldots, d .
\end{gathered}
$$

The existence of such vectors follows from the fact that the matrix with components $\left\langle\Lambda_{M} \psi^{(j)}, \psi^{(k)}\right\rangle, j, k=1, \ldots, d$, is non-singular (real symmetric and positive definite). In a similar way, we can choose a basis $\left\{\psi^{(d+1)}, \ldots, \psi^{(m)}\right\}$ of $\Lambda_{M}^{-1} \mathscr{M}^{\perp}$ satisfying (2.15) for $j, k=d+1, \ldots, m$. It is easy to see that both $\left\{\psi^{(1)}, \ldots, \psi^{(m)}\right\}$ and $\left\{\phi^{(1)}, \ldots, \phi^{(m)}\right\}$ are bases of $\mathbb{R}^{m}$ and satisfy $(2.15)$ for $j, k=1, \ldots, m$, namely, they are dual bases to each other.

\section{Global Existence and Decay of Solutions}

We consider the initial value problem (1.5), (1.6) in a neighborhood of an absolutely Maxwellian. We assume the following

Condition 1. Let $\psi \in \mathscr{M}$ and $\lambda \psi=V \psi$ for $\lambda \in \mathbb{R}$. Then it follows $\psi=0$.

Several conditions each of which is equivalent to Condition 1 were given in [17]. For the details, see Theorem 3.2 below. Under Condition 1 the problem (1.5), (1.6) is solved globally in time as follows.

Theorem 3.1 ([13,17]) Assume Condition 1. Let $M$ be an absolute Maxwellian with positive components. (i) Suppose that $F_{0}-M \in H^{s}, s \geqq 1$, and $\left\|F_{0}-M\right\|_{s}$ is small. Then the problem (1.5), (1.6) has a unique global solution $F(t, x)$ satisfying $F-$ $M \in C^{0}\left([0, \infty] ; H^{s}\right) \cap C^{1}\left([0, \infty] ; H^{s-1}\right)$. Moreover, $F(t, x)$ converges to $M$ in $\mathscr{B}^{s-1}$ norm as $t \rightarrow \infty$. (ii) If $F_{0}-M \in H^{s} \cap L^{1}, s \geqq 1$, and $E_{s}=\left\|F_{0}-M\right\|_{s}+\left\|F_{0}-M\right\|_{L^{1}}$ is small, then the solution $F(t, x)$ in (i) satisfies

$$
\left\|\partial_{x}^{l}(F(t)-M)\right\|_{s-1} \leqq C E_{s}(1+t)^{-(1 / 2+l) / 2}
$$

for $t \in[0, \infty)$, where $0 \leqq l \leqq s$ and $C$ is a constant. 
The estimate (3.1) with $1 \leqq l \leqq s$ was not proved in the previous papers $[13,17]$, and therefore we will give its proof after some preparations. We first derive the linearized equation of (1.5) around the absolute Maxwellian M. Put

$$
F=M+\Lambda_{M}^{1 / 2} f .
$$

Substituting (3.2) into (1.5), we obtain

$$
f_{t}+V f_{x}+L_{M} f=\Gamma_{M}(f, f),
$$

where $L_{M}$ and $\Gamma_{M}$ are defined by (2.11) and (2.12), respectively. The corresponding linearized equation is

$$
f_{t}+V f_{x}+L_{M} f=0 .
$$

The eigenvalue problem associated with (3.4) is

$$
\mu \zeta=B(i \xi) \zeta, \quad B(i \xi)=-\left(L_{M}+i \xi V\right), \quad \xi \in \mathbb{R},
$$

where $\mu \in \mathbb{C}$ and $\zeta \in \mathbb{C}^{m}$. We denote the eigenvalues of (3.5) by $\mu=\mu_{M}(i \xi)$. The following result obtained in [17] plays a crucial role in the study of the global existence problem for (1.5).

Theorem 3.2. ([17]) (i) Let $M$ be an absolute Maxwellian. Then the following four conditions are equivalent.

$$
\begin{gathered}
\text { Let } L_{M} \zeta=0 \text { and } \lambda \zeta=V \zeta \text { for } \lambda \in \mathbb{R} \text { and } \zeta \in \mathbb{R}^{m} . \\
\text { Then it follows } \zeta=0 \text {. } \\
\operatorname{Re} \mu_{M}(i \xi)<0 \text { for any } \xi \neq 0 .
\end{gathered}
$$

There is a positive constant $c_{M}$ such that $\operatorname{Re} \mu_{M}(i \xi) \leqq-c_{M} \rho(\xi)$

$$
\text { for any } \xi \in \mathbb{R} \text {, where } \rho(\xi)=\xi^{2} /\left(1+\xi^{2}\right) \text {. }
$$

There exists an $m \times m$ real skew-symmetric matrix $K_{M}$ such that the symmetric part of $K_{M} V+L_{M}$ is positive definite.

ii) If Condition 1 is assumed, then (3.6) $-(3.6)_{4}$ hold for any absolute Maxwellian $M$. Conversely, if one of $(3.6)_{1}-(3.6)_{4}$ is true for an absolute Maxwellian $M$, then Condition 1 is satisfied.

We denote by $e^{t B}$ the semigroup of the linearized Eq. (3.4). When the matrix exponential $e^{t B(i \xi)}$ is well defined, we get the relation

$$
\left(e^{t B} f\right)^{\wedge}(\xi)=e^{t B(i \xi)} \hat{f}(\xi)
$$

where $\hat{f}(\xi)$ denotes the Fourier transform of $f(x)$. If Condition 1 (or equivalently, $\left.(3.6)_{4}\right)$ is assumed, we have

$$
\left|\left(e^{t B} f\right)^{\wedge}(\xi)\right| \leqq C e^{-c \rho(\xi) t}|\hat{f}(\xi)|, \quad \xi \in \mathbb{R}
$$

where $\rho(\xi)=\xi^{2} /\left(1+\xi^{2}\right), C$ and $c$ are positive constants. For the proof of (3.8), see [13 or 19]. From (3.8) we easily obtain

$$
\left\|\partial_{x}^{l}\left(e^{t B} f\right)\right\| \leqq C e^{-c t}\left\|\partial_{x}^{l} f\right\|+C(1+t)^{-(1 / 2+l-k) / 2}\left\|\partial_{x}^{k} f\right\|_{L^{1}},
$$


where $0 \leqq k \leqq l, C$ and $c$ are positive constants, and $f(x)$ is a function such that the norms on the right-hand side of (3.9) are finite. It is also known ([13]) that if $f(x) \in N\left(L_{M}\right)^{\perp}$ holds for any $x \in \mathbb{R}$, then (3.9) can be improved to

$$
\left\|\partial_{x}^{l}\left(e^{t B} f\right)\right\| \leqq C e^{-c t}\left\|\partial_{x}^{l} f\right\|+C(1+t)^{-(3 / 2+l-k) / 2}\left\|\partial_{x}^{k} f\right\|_{L^{1}},
$$

where $0 \leqq k \leqq l+1$ and $l \geqq 0$.

Proof of the Estimate (3.1). Let $F(t, x)$ be the solution of (1.5), (1.6) in (i) of Theorem 3.1. The function $f(t, x)$ defined by (3.2) satisfies (3.3). Therefore, using the semigroup $e^{t B}$, we get the formula

$$
f(t)=e^{t B} f_{0}+\int_{0}^{t} e^{(t-\tau) B} \Gamma_{M}(f, f)(\tau) d \tau
$$

where $f_{0}(x)=\Lambda_{M}^{-1 / 2}\left(F_{0}(x)-M\right)$. Put

$$
M_{l}(t)=\sup _{0 \leqq \tau \leqq t}(1+\tau)^{(1 / 2+l) / 2}\left\|\partial_{x}^{l} f(\tau)\right\|_{s-l}
$$

for $0 \leqq l \leqq s$. It suffices to get the estimate $M_{l}(t) \leqq C E_{s}$ with a constant $C$. We apply $\partial_{x}^{j}, 0 \leqq j \leqq s$, to (3.11) and estimate each equation by using (3.9) and (3.10). (Recall that $\Gamma_{M}(f, f) \in N\left(L_{M}\right)^{\perp}$ for any $\left.f \in \mathbb{R}^{m}\right)$. Summing up for $0 \leqq j \leqq s$, we obtain

$$
\begin{aligned}
\|f(t)\|_{s} \leqq & C E_{s}(1+t)^{-1 / 4}+C \int_{0}^{t} e^{-c(t-\tau)}\left\|\Gamma_{M}(f, f)(\tau)\right\|_{s} d \tau \\
& +C \int_{0}^{t}(1+t-\tau)^{-3 / 4}\left\|\Gamma_{M}(f, f)(\tau)\right\|_{L^{1}} d \tau .
\end{aligned}
$$

By the definition of $M_{0}(t)$, the middle and the last terms on the right-hand side of (3.13) are majorized by

$$
\begin{aligned}
& C M_{0}(t)^{2} \int_{0}^{t} e^{-c(t-\tau)}(1+\tau)^{-1 / 2} d \tau \leqq C M_{0}(t)^{2}(1+t)^{-1 / 2} \\
& C M_{0}(t)^{2} \int_{0}^{t}(1+t-\tau)^{-3 / 4}(1+\tau)^{-1 / 2} d \tau \leqq C M_{0}(t)^{2}(1+t)^{-1 / 4}
\end{aligned}
$$

respectively. Therefore we arrive at the inequality $M_{0}(t) \leqq C E_{s}+C M_{0}(t)^{2}$, from which follows the desired estimate $M_{0}(t) \leqq C E_{s}$ if $E_{s}$ is small. Thus the proof of (3.1) with $l=0$ is completed.

Next we show (3.1) with $l=1$. From the estimates for the derivatives $\partial_{x}^{j} f, 1 \leqq$ $j \leqq s$, we obtain

$$
\begin{aligned}
& \left\|\partial_{x} f(t)\right\|_{s-1} \leqq C E_{s}(1+t)^{-3 / 4}+C \int_{0}^{t} e^{-c(t-\tau)} . \\
& \left\|\partial_{x} \Gamma_{M}(f, f)(\tau)\right\|_{s-1} d \tau+C \int_{0}^{t / 2}(1+t-\tau)^{-5 / 4}\left\|\Gamma_{M}(f, f)(\tau)\right\|_{L^{1}} d \tau \\
& \quad+C \int_{t / 2}^{t}(1+t-\tau)^{-3 / 4}\left\|\partial_{x} \Gamma_{M}(f, f)(\tau)\right\|_{L^{1}} d \tau .
\end{aligned}
$$

Here we have taken $k$ in (3.10) such that $k=0$ on $[0, t / 2]$ and $k=1$ on $[t / 2, t]$. We 
estimate each term on the right-hand side of (3.14) by using (3.1) with $l=0$ and the definition of $M_{1}(t)$. We know that the second term is majorized by

$$
C E_{s} M_{1}(t) \int_{0}^{t} e^{-c(t-\tau)}(1+\tau)^{-1} d \tau \leqq C E_{s} M_{1}(t)(1+t)^{-1} .
$$

The third and the last terms are estimated respectively by

$$
\begin{gathered}
C E_{s}^{2} \int_{0}^{t / 2}(1+t-\tau)^{-5 / 4}(1+\tau)^{-1 / 2} d \tau \leqq C E_{s}^{2}(1+t)^{-3 / 4} \\
C E_{s} M_{1}(t) \int_{t / 2}^{t}(1+t-\tau)^{-3 / 4}(1+\tau)^{-1} d \tau \leqq C E_{s} M_{1}(t)(1+t)^{-3 / 4} .
\end{gathered}
$$

Combining these estimates, we obtain the inequality $M_{1}(t) \leqq C E_{s}+C E_{s} M_{1}(t)$, which gives $M_{1}(t) \leqq C E_{\mathrm{s}}$ if $E_{\mathrm{s}}$ is small. Thus (3.1) is proved for $l=1$. The estimates for higher derivatives are shown in the same way by using the induction for $l$. We omit the details.

\section{The Euler Equation}

Following [8], we derive the Euler equation as the first approximation of the Chapman-Enskog expansion to (1.5). We assume that the solution $F$ of (1.5) has the expansion

$$
F=\sum_{n=0}^{\infty} F^{(n)}
$$

Let $w={ }^{t}\left(w_{1}, \ldots, w_{d}\right)$ be the moments of $F$ defined by (2.4). Each $F^{(n)}$ is assumed to be a function depending only on $\partial_{x}^{\alpha} w(\alpha \geqq 0)$ such that $F^{(n)} \in \mathscr{M}^{\perp}$ for $n \geqq 1$. Consequently, we have

$$
\begin{gathered}
w_{j}=\left\langle F^{(0)}, \psi^{(j)}\right\rangle, \quad j=1, \ldots, d, \\
\left\langle F^{(n)}, \psi^{(j)}\right\rangle=0, \quad j=1, \ldots, d, \quad n \geqq 1 .
\end{gathered}
$$

Taking the inner product of $(1.5)$ with $\psi^{(j)}, j=1, \ldots, d$, we obtain

$$
\left(w_{j}\right)_{t}+\left(\left\langle V F, \psi^{(j)}\right\rangle\right)_{x}=0, \quad j=1, \ldots, d .
$$

Each $F^{(n)}$ is determined successively by the equations

$$
\begin{gathered}
Q\left(F^{(0)}, F^{(0)}\right)=0, \\
2 Q\left(F^{(0)}, F^{(n)}\right)=-\sum_{k=1}^{n-1} Q\left(F^{(k)}, F^{(n-k)}\right)+V F_{x}^{(n-1)} \\
+\sum_{k+l=n-1} \sum_{j=1}^{d} \sum_{\alpha=0}^{\infty} \frac{\partial F^{(k)}}{\partial\left(\partial_{x}^{\alpha} w_{j}\right)} \partial_{x}^{\alpha}\left(-\left\langle V F^{(l)}, \psi^{(j)}\right\rangle\right)_{x}, \quad n \geqq 1,
\end{gathered}
$$

supplemented by the conditions $(4.2)_{0}$ and $(4.2)_{n}, n \geqq 1$. The Euler equation is Eq. (4.3) for $F=F^{(0)}$, where $F^{(0)}$ is determined by $(4.4)_{0}$ and $(4.2)_{0}$. By Lemma 2.1 (see (2.7), (2.8)), we have the expressions $F^{(0)}=\bar{F}(u)$ and

$$
w=w(u)={ }^{t}\left(\left\langle\bar{F}(u), \psi^{(j)}\right\rangle\right)_{1 \leqq I \leqq d},
$$


and hence $F^{(0)}=\bar{F}(u(w))$, where $u=u(w)$ is the inverse mapping of $w=w(u)$. Here all the components of $F^{(0)}$ are assumed to be positive. Consequently, (4.3) for $F=F^{(0)}$ takes the form

where

$$
w(u)_{t}+h(u)_{x}=0 \quad \text { or } \quad A^{0}(u) u_{t}+A(u) u_{x}=0
$$

$$
\begin{aligned}
h(u) & ={ }^{t}\left(\left\langle V \bar{F}(u), \psi^{(j)}\right\rangle\right)_{1 \leqq J \leqq d}, \\
A^{0}(u) & =D_{u} w(u)=\left(\left\langle\Lambda_{\bar{F}(u)} \psi^{(j)}, \psi^{(k)}\right\rangle\right)_{1 \leqq j, k \leqq d}, \\
A(u) & =D_{u} h(u)=\left(\left\langle V \Lambda_{\bar{F}(u)} \psi^{(j)}, \psi^{(k)}\right\rangle\right)_{1 \leqq J, k \leqq d} .
\end{aligned}
$$

Here $D_{u}$ denotes the differentiation with respect to $u$. Also, (4.6) is rewritten in the form

$$
w_{t}+\tilde{h}(w)_{x}=0 \quad \text { or } \quad w_{t}+\tilde{A}(w) w_{x}=0
$$

where

$$
\tilde{h}(w)=h(u(w)), \quad \tilde{A}(w)=D_{w} \tilde{h}(w) .
$$

The Euler equation (4.8) is regarded as a symmetric hyperbolic system because $A^{0}(u)$ and $A(u)$ are real symmetric and in addition $A^{0}(u)$ is positive definite. Notice that

$$
\widetilde{A}(w(u))=A(u) A^{0}(u)^{-1}={ }^{t} \hat{A}(u), \quad \hat{A}(u)=A^{0}(u)^{-1} A(u) .
$$

In what follows we assume the following

Condition 2. The Euler equation (4.8) is strictly hyperbolic and each characteristic field is either genuinely nonlinear or linearly degenerate in the sense of Lax [15].

This condition means that for each $w$, the matrix $\tilde{A}(w)$ has real and distinct eigenvalues

$$
\lambda_{1}(w)<\cdots<\lambda_{d}(w)
$$

and for each $j=1, \ldots, d$, we have either $\left\langle\nabla_{w} \lambda_{j}(w), \tilde{r}_{j}(w)\right\rangle \neq 0$ (genuinely nonlinear) or $\left\langle\nabla_{w} \lambda_{j}(w), \tilde{r}_{j}(w)\right\rangle=0$ (linearly degenerate) for all $w$. Here $\tilde{r}_{j}(w)$ is the right eigenvector of $\tilde{A}(w)$ for $\lambda_{j}(w), \nabla_{w}$ denotes the gradient with respect to $w$ and $\langle$,$\rangle the$ standard inner product in $\mathbb{R}^{d}$. Without loss of generality we may assume that either

$$
\left\langle\nabla_{w} \lambda_{j}(w), \tilde{r}_{j}(w)\right\rangle=1 \quad \text { or }\left\langle\nabla_{w} \lambda_{j}(w), \tilde{r}_{j}(w)\right\rangle=0
$$

for all $w$. It is easily seen that $\left\langle A^{0}(u(w))^{-1} \tilde{r}_{j}(w), \tilde{r}_{k}(w)\right\rangle=0$ for $j \neq k$. Therefore,

$$
\left\langle\tilde{l}_{j}(w), \tilde{r}_{k}(w)\right\rangle=\delta_{j k}, \quad j, k=1, \ldots, d,
$$

where

$$
\begin{aligned}
& \tilde{l}_{j}(w)=c_{j}(w)^{t} \tilde{r}_{j}(w) A^{0}(u(w))^{-1}, \\
& c_{j}(w)=\left\langle A^{0}(u(w))^{-1} \tilde{r}_{j}(w), \tilde{r}_{j}(w)\right\rangle^{-1}>0 .
\end{aligned}
$$

Notice that $\tilde{l}_{j}(w)$, a row vector in $\mathbb{R}^{d}$, is the left eigenvector of $\widetilde{A}(w)$ for $\lambda_{j}(w)$. We have the spectral resolution

$$
\widetilde{A}(w)=\sum_{j=1}^{d} \lambda_{j}(w) \widetilde{P}_{j}(w), \quad \widetilde{P}_{j}(w)=\tilde{r}_{j}(w) \widetilde{l}_{j}(w) .
$$


From (4.10) we know that $\lambda_{j}(w(u))$ are the eigenvalues of $\hat{A}(u)$. The corresponding right and left eigenvectors, $\hat{r}_{j}(u)$ and $\hat{l}_{j}(u)$, are given respectively by

$$
\begin{aligned}
& \hat{r}_{j}(u)=c_{j}(w(u))^{-1 / 2 t} \tilde{l}_{j}(w(u))=c_{j}(w(u))^{1 / 2} A^{0}(u)^{-1} \tilde{r}_{j}(w(u)), \\
& \hat{l}_{j}(u)=c_{j}(w(u))^{1 / 2 t} \tilde{r}_{j}(w(u))=c_{j}(w(u))^{-1 / 2} \widetilde{l}_{j}(w(u)) A^{0}(u) .
\end{aligned}
$$

Here $\hat{r}_{j}(u)$ and $\hat{l}_{j}(u)$ are chosen so as to satisfy the normalizations

$$
\begin{aligned}
\left\langle A^{0}(u) \hat{r}_{j}(u), \hat{r}_{k}(u)\right\rangle & =\delta_{j k}, \\
\left\langle\hat{l}_{j}(u), \hat{r}_{k}(u)\right\rangle & =\delta_{j k}, \quad j, k=1, \ldots, d .
\end{aligned}
$$

\section{Spectral Representation}

We consider the eigenvalue problem (3.5). Assymptotic expansions for $\xi \rightarrow 0$ of eigenvalues and eigenvectors were given in [7]. Our aim is to determine the coefficients appearing in the expansions by using Conditions 1 and 2 .

Let $M$ be an absolute Maxwellian with positive components. We denote the moments of $M$ by $\bar{w}={ }^{t}\left(\bar{w}_{1}, \ldots, \bar{w}_{d}\right)$ and put $\bar{u}=u(\bar{w})$. We apply the perturbation theory for matrices (see [11]) to the problem (3.5) to obtain the asymptotic expansions of $\mu=\mu(i \xi)$ and $\zeta=\zeta(i \xi)$ for $\xi \rightarrow 0$ :

$$
\mu(i \xi)=\sum_{n=0}^{\infty}(i \xi)^{n} \mu^{(n)}, \quad \zeta(i \xi)=\sum_{n=0}^{\infty}(i \xi)^{n} \zeta^{(n)},
$$

where $\mu^{(n)} \in \mathbb{R}$ and $\zeta^{(n)} \in \mathbb{R}^{m}$ because $L_{M}$ and $V$ are real symmetric. Substitution of (5.1) into (3.5) yields

$$
\begin{gathered}
\mu^{(0)} \zeta^{(0)}+L_{M} \zeta^{(0)}=0, \\
\sum_{l=0}^{n} \mu^{(l)} \zeta^{(n-l)}+L_{M} \zeta^{(n)}+V \zeta^{(n-1)}=0, \quad n \geqq 1 .
\end{gathered}
$$

Equation $(5.2)_{0}$ implies that $-\mu^{(0)}$ is an eigenvalue of $L_{M}$ and $\zeta^{(0)}$ is the corresponding right eigenvector. Therefore we have $\mu^{(0)}=0$ (with multiplicity $d$ ) or $\mu^{(0)}<0$ by Lemma 2.2. We treat the case

$$
\mu^{(0)}=0
$$

In this case we have $\zeta^{(0)} \in N\left(L_{M}\right)$, and therefore

$$
\zeta^{(0)}=\sum_{k=1}^{d} \beta_{k} \Lambda_{M}^{1 / 2} \psi^{(k)}
$$

by (2.13), where $\beta_{1}, \ldots, \beta_{d}$ are real constants. Substituting $(5.3)_{0}$ and $(5.4)_{0}$ into $(5.2)_{1}$ and taking the inner product (in $\mathbb{R}^{m}$ ) with $\Lambda_{M}^{1 / 2} \psi^{(k)}, k=1, \ldots, d$, we obtain $\mu^{(1)} A^{0}(\bar{u}) \beta+A(\bar{u}) \beta=0$, where $\beta={ }^{t}\left(\beta_{1}, \ldots, \beta_{d}\right)$. Here we used (4.7). Therefore, $-\mu^{(1)}$ is an eigenvalue of $\hat{A}(\bar{u})=A^{0}(\bar{u})^{-1} A(\bar{u})$ and $\beta$ is the corresponding right eigenvector. Thus we have

$$
\mu^{(1)}=-\lambda_{j}(\bar{w})
$$

and $\beta=\hat{r}_{j}(\bar{u})$ for $j=1, \ldots, d$. Consequently, we have from $(5.4)_{0}$, 


$$
\zeta^{(0)}=e_{j} \equiv \sum_{k=1}^{d} \hat{r}_{j k}(\bar{u}) \Lambda_{M}^{1 / 2} \psi^{(k)}
$$

where $\hat{r}_{j k}(\bar{u})$ is the $k$-th component of $\hat{r}_{j}(\bar{u})$. Here $e_{j}$ are normalized so as to satisfy (by (4.18))

$$
\left\langle e_{j}, e_{k}\right\rangle=\delta_{j k}, \quad j, k=1, \ldots, d .
$$

Notice that $\left\{e_{1}, \ldots, e_{d}\right\}$ is an orthonormal basis of $N\left(L_{M}\right)$. Substituting $(5.3)_{0,1}$ and $(5.4)_{0}^{\prime}$ into $(5.2)_{1}$, we get $L_{M} \zeta^{(1)}=\left(\lambda_{j}(\bar{w})-V\right) e_{j}$. This equation can be solved (non-uniquely) for $\zeta^{(1)}$ because

$$
\left(\lambda_{j}(\bar{w})-V\right) e_{j} \in N\left(L_{M}\right)^{\perp}
$$

we have

$$
\zeta^{(1)}=e_{j}^{(1)} \quad \text { with } \quad e_{j}^{(1)}=L_{M}^{-1}\left(\lambda_{j}(\bar{w})-V\right) e_{j}+P_{0} e_{j}^{(1)}
$$

where $P_{0}$ denotes the orthogonal projection onto $N\left(L_{M}\right)$, namely, $P_{0} f=\sum_{k=0}^{d}$ $\left\langle f, e_{k}\right\rangle e_{k}$ for $f \in \mathbb{R}^{m}$. Substituting $(5.3)_{0,1},(5.4)_{0}^{\prime}$ and $(5.4)_{1}$ into $(5.2)_{2}$ and taking the inner product with $e_{j}$, we get the formula

$$
\mu^{(2)}=\kappa_{j} \equiv\left\langle L_{M}^{-1}\left(\lambda_{j}(\bar{w})-V\right) e_{j},\left(\lambda_{j}(\bar{w})-V\right) e_{j}\right\rangle,
$$

where (5.5) and (5.6) were used. Since $L_{M} e_{j}=0$ and $e_{j} \neq 0$, we conclude by Condition 1 (or equivalently, $\left.(3.6)_{1}\right)$ that $\left(\lambda_{j}(\bar{w})-V\right) e_{j} \neq 0$. Therefore we have $\kappa_{j}>0$ because $L_{M}^{-1}$ is real symmetric and positive definite on $N\left(L_{M}\right)^{\perp}$. These considerations are summarized in the following lemma.

Lemma 5.1. Assume Conditions 1 and 2 and consider the problem (3.5). Then there is a positive constant $\delta$ such that for $|\xi|<\delta$, we have d eigenvalues $\mu_{j}=\mu_{j}(i \xi)$, $j=1, \ldots, d$, which tend to zero as $\xi \rightarrow 0$. We denote the corresponding eigenvectors by $\zeta_{j}=\zeta_{j}(i \xi), j=1, \ldots, d$. We have the Taylor series expansions for $|\xi|<\delta$ :

$$
\mu_{j}(i \xi)=\sum_{n=1}^{\infty}(i \xi)^{n} \mu_{j}^{(n)}, \quad \zeta_{j}(i \xi)=\sum_{n=0}^{\infty}(i \xi)^{n} \zeta_{j}^{(n)}
$$

where $\mu_{j}^{(n)} \in \mathbb{R}$ and $\zeta_{j}^{(n)} \in \mathbb{R}^{m}$. In particular, we have

$$
\begin{aligned}
& \mu_{j}^{(1)}=-\lambda_{j}(\bar{w}), \quad \mu_{j}^{(2)}=\kappa_{j}(\bar{w})>0, \\
& \zeta_{j}^{(0)}=e_{j}(\bar{w}), \quad \zeta_{j}^{(1)}=e_{j}^{(1)}(\bar{w}) .
\end{aligned}
$$

For explicit forms of these coefficients, see $(5.4)_{0}^{\prime},(5.4)_{1}$ and $(5.3)_{2}$. The eigenvectors $\zeta_{j}(i \xi)^{\prime}$ can be normalized by

$$
\left(\zeta_{j}(i \xi), \zeta_{k}(-i \xi)\right)=\delta_{j k}, \quad j, k=1, \ldots, d,
$$

where (,) denotes the standard inner product in $\mathbb{C}^{m}$.

By this lemma, the matrix exponential $e^{t B(i \xi)}$ is well defined for $|\xi|<\delta$ and has the following expression.

$$
e^{t B(i \xi)} f=\sum_{j=1}^{d} e^{t \mu_{j}(i \xi)}\left(f, \zeta_{j}(-i \xi)\right) \zeta_{j}(i \xi)+Z(t, i \xi) f
$$


for $f \in \mathbb{C}^{m}$. Here $Z(t, i \xi)$ is the matrix satisfying

$$
|Z(t, i \xi)| \leqq C e^{-c t}, \quad|\xi|<\delta,
$$

where $C$ and $c$ are positive constants.

\section{Approximation by Uniformly Parabolic System, I}

Let $F(t, x)$ be the solution of (1.5), (1.6) constructed in Theorem 3.1 and let $f(t, x)$ be the function defined by (3.2). We have the formula (3.11), or in the Fourier transform,

$$
\hat{f}(t, \xi)=\left(e^{t B} f_{0}\right) \hat{\gamma}(\xi)+\int_{0}^{t}\left(e^{(t-\tau) B} \Gamma_{M}(f, f) \hat{)}(\tau, \xi) d \tau,\right.
$$

where $f_{0}(x)=\Lambda_{M}^{-1 / 2}\left(F_{0}(x)-M\right)$. For $|\xi|<\delta$, the matrix exponential $e^{t B(i \xi)}$ is well defined and hence (3.7) holds true. Therefore, using (5.10), we obtain

$$
\begin{aligned}
\hat{f}(t, \xi)= & \sum_{j=1}^{d} e^{t \mu_{J}(i \xi)}\left(\hat{f}_{0}(\xi), \zeta_{j}(-i \xi)\right) \zeta_{j}(i \xi)+Z(t, i \xi) \hat{f}_{0}(\xi) \\
& +\int_{0}^{t} \sum_{j=1}^{d} e^{(t-\tau) \mu_{j}(i \xi)}\left(\hat{\Gamma}_{M}(f, f)(\tau, \xi), \zeta_{j}(-i \xi)\right) \zeta_{j}(i \xi) d \tau \\
& +\int_{0}^{t} Z(t-\tau, i \xi) \hat{\Gamma}_{M}(f, f)(\tau, \xi) d \tau, \quad|\xi|<\delta
\end{aligned}
$$

Taking (6.2) into account, we define $G(t, x)$ by

$$
\begin{aligned}
G(t, x)= & M+\Lambda_{M}^{1 / 2} g(t, x), \\
\hat{g}(t, \xi)= & \sum_{j=1}^{d} e^{t v_{j}(i \xi)}\left(\hat{f}_{0}(\xi), e_{j}\right) e_{j} \\
& +\int_{0}^{t} \sum_{j=1}^{d} e^{(t-\tau) v_{j}(i \xi)}\left(\hat{\Gamma}_{M}(g, g)(\tau, \xi),-i \xi e_{j}^{(1)}\right) e_{j} d \tau,
\end{aligned}
$$

where

$$
v_{j}(i \xi)=-i \xi \lambda_{j}(\bar{w})+(i \xi)^{2} \kappa_{j}(\bar{w}), \quad j=1, \ldots, d .
$$

(Here $\lambda_{j}(\bar{w}), \kappa_{j}(\bar{w}), e_{j}=e_{j}(\bar{w})$ and $e_{j}^{(1)}=e_{j}^{(1)}(\bar{w})$ are the coefficients in (5.8).) We shall show that $G(t, x)$ is well defined for all $(\mathrm{t}, x)$ and gives an approximation to $F(t, x)$ for $t \rightarrow \infty$. For this purpose, we first derive the equation of $g(t, x)$. Substituting $(5.4)_{1}$ into (6.4) and using (5.6) and the fact that $\Gamma_{M}(g, g)$ is perpendicular to $N\left(L_{M}\right)$ (by Lemma 2.2), we have

$$
\begin{aligned}
\hat{g}(t, \xi)= & \sum_{j=1}^{d} e^{t v_{J}(i \xi)}\left(\hat{f}_{0}(\xi), e_{j}\right) e_{j} \\
& -\int_{0}^{t} i \xi \sum_{j=1}^{d} e^{(t-\tau) v_{j}(i \xi)}\left(V L_{M}^{-1} \hat{\Gamma}_{M}(g, g)(\tau, \xi), e_{j}\right) e_{j} d \tau .
\end{aligned}
$$

This expression implies that $g(t, x) \in N\left(L_{M}\right)$, and hence $G(t, x)-M \in \Lambda_{M} \mathscr{M}$ for all 
$(t, x)$. Consequently, we have the expressions

$$
\begin{gathered}
g(t, x)=\sum_{j=1}^{d} g^{j}(t, x) e_{j}, \\
G(t, x)-M=\sum_{j=1}^{d}\left(z_{j}(t, x)-\bar{w}_{j}\right) \phi^{(j)},
\end{gathered}
$$

where $g^{j}(t, x)=\left\langle g(t, x), e_{j}\right\rangle$ and $z_{j}(t, x)=\left\langle G(t, x), \psi^{(j)}\right\rangle, j=1, \ldots, d$. Here we used (5.5) and (2.15). Note that $z_{j}(t, x)$ and $\bar{w}_{j}$ are the $j$-th moments of $G(t, x)$ and $M$, respectively. Take the inner product (in $\mathbb{C}^{m}$ ) of $(6.6)$ with $e_{j}, j=1, \ldots, d$, and differentiate the both sides with respect to $t$. We substitute (6.5) and (6.7) into the resulting equation and then take the inverse Fourier transform to obtain

$$
\begin{gathered}
g_{t}^{j}+\lambda_{j}(\bar{w}) g_{x}^{j}+\sum_{k, l=1}^{d} q_{k l}^{j}(\bar{w})\left(g^{k} g^{l}\right)_{x}=\kappa_{j}(\bar{w}) g_{x x}^{j}, \\
g^{j}(0, x)=\left\langle F_{0}(x)-M, \Lambda_{M}^{-1 / 2} e_{j}\right\rangle, \quad j=1, \ldots, d,
\end{gathered}
$$

where

$$
q_{k l}^{j}(\bar{w})=\left\langle V L_{M}^{-1} \Gamma_{M}\left(e_{k}, e_{l}\right), e_{j}\right\rangle, \quad j, k, l=1, \ldots, d .
$$

Note that (6.9) is a semilinear uniformly parabolic system because $\kappa_{j}(\bar{w})>0$ for $j=1, \ldots, d$.

We rewrite (6.9) into the equation of the moments $z_{j}=z_{j}(t, x), j=1, \ldots, d$, of $G(t, x)$. Put $z={ }^{t}\left(z_{1}, \ldots, z_{d}\right)$. A simple calculation shows that

$$
g^{j}=\left\langle z-\bar{w}, \hat{r}_{j}(\bar{u})\right\rangle=c_{j}(\bar{w})^{-1 / 2}\left\langle z-\bar{w}, \tilde{l}_{j}(\bar{w})\right\rangle
$$

for $j=1, \ldots, d$. Here we used $(5.4)_{0}^{\prime}$ and (4.17). These relations together with (4.18) and (4.17) yield

$$
z-\bar{w}=\sum_{j=1}^{d} g^{j t} \hat{l}_{j}(\bar{u})=\sum_{j=1}^{d} g^{j} c_{j}(\bar{w})^{1 / 2} \tilde{r}_{j}(\bar{w}) .
$$

From (6.9), (6.10), (6.12) and (6.13) we easily obtain

where

$$
\begin{aligned}
z_{t}+k(z)_{x} & =\widetilde{D}(\bar{w}) z_{x x} \\
z(0, x) & =w_{0}(x) \equiv{ }^{t}\left(\left\langle F_{0}(x), \psi^{(j)}\right\rangle\right)_{1 \leqq j \leqq d},
\end{aligned}
$$

$$
\begin{aligned}
k(z) & =\tilde{h}(\bar{w})+\tilde{A}(\bar{w})(z-\bar{w})+q(z), \\
\tilde{D}(\bar{w}) & =\sum_{j=1}^{d} \kappa_{j}(\bar{w}) \widetilde{P}_{j}(\bar{w}),
\end{aligned}
$$

and $q(z)$ is the quadratic function of $z-\bar{w}$ :

$$
\begin{aligned}
q(z) & =\sum_{i, j, k=1}^{d} Q_{j k}^{i}(\bar{w})\left\langle z-\bar{w}, \tilde{l}_{j}(\bar{w})\right\rangle\left\langle z-\bar{w}, \widetilde{l}_{k}(\bar{w})\right\rangle \tilde{r}_{i}(\bar{w}), \\
Q_{j k}^{i}(\bar{w}) & =q_{j k}^{i}(\bar{w})\left(c_{i}(\bar{w}) / c_{j}(\bar{w}) c_{k}(\bar{w})\right)^{1 / 2} .
\end{aligned}
$$

In deriving (6.16), we used the spectral resolution (4.16). The system (6.14) is also 
semilinear and uniformly parabolic and therefore the problem (6.14), (6.15) can be solved globally in time, provided that the initial data $w_{0}(x)$ are close to the constant state $\bar{w}$. (See, for example, Proposition 6.1 of [14].)

To obtain a decay estimate of solutions, we consider the linearized system of (6.14) around $z=\bar{w}$,

$$
z_{t}+\tilde{A}(\bar{w}) z_{x}=\tilde{D}(\bar{w}) z_{x x} .
$$

We denote the semigroup of (6.18) by $e^{t S}$. Then we have $\left(e^{t S} z\right)^{\wedge}(\xi)=e^{t S(i \xi)} \hat{z}(\xi)$, where

$$
\begin{aligned}
S(i \xi) & =-i \xi \tilde{A}(\bar{w})+(i \xi)^{2} \widetilde{D}(w)=\sum_{j=1}^{d} v_{j}\left(i \dot{\zeta}_{j}\right) \widetilde{P}_{j}(\bar{w}), \\
e^{t S(i \xi)} & =\sum_{j=1}^{d} e^{t v_{j}(i \xi)} \widetilde{P}_{j}(\bar{w}) .
\end{aligned}
$$

Recall that $v_{j}(i \xi)$ is given by (6.5). By virtue of the spectral resolution (6.20), we easily obtain

$$
\left\|\partial_{x}^{l}\left(e^{t S} z\right)\right\| \leqq C e^{-c t}\left\|\partial_{x}^{l} z\right\|+C(1+t)^{-(1 / 2+l-k) / 2}\left\|\partial_{x}^{k} z\right\|_{L^{1}}
$$

where $0 \leqq k \leqq l, C$ and $c$ are positive constants. We also have

$$
\begin{aligned}
\left\|\partial_{x}^{l} \int_{0}^{t} e^{(t-\tau) S} z(\tau) d \tau\right\| \leqq & C\left(\int_{0}^{t} e^{-c(t-\tau)}\left\|\partial_{x}^{l-1} z(\tau)\right\|^{2} d \tau\right)^{1 / 2} \\
& +C \int_{0}^{t}(1+t-\tau)^{-(1 / 2+l-k) / 2}\left\|\partial_{x}^{k} z(\tau)\right\|_{L^{1}} d \tau
\end{aligned}
$$

where $l \geqq 1$ and $0 \leqq k \leqq l$. Using (6.21) and (6.22), we can show the decay of solutions of (6.14), (6.15) in the same way as in the proof of (3.1). Summarizing all the considerations, we have

Proposition 6.1. Assume Conditions 1 and 2. We denote by $w_{0}(x)$ the moments of $F_{0}(x)$, i.e., $w_{0}(x)={ }^{i}\left(\left\langle F_{0}(x), \psi^{(j)}\right\rangle_{1 \leqq J \leqq d}\right.$. (i) If $w_{0}-\bar{w} \in H^{s}, s \geqq 1$, and $\left\|w_{0}-\bar{w}\right\|_{s}$ is small, then the problem (6.14), (6.15) has a unique global solution $z(t, x)$ satisfying $z-\bar{w} \in C^{0}\left([0, \infty) ; H^{s}\right)\left(\cap C^{1}\left([0, \infty) ; H^{s-2}\right)\right.$ if $\left.s \geqq 2\right)$ and $\partial_{x} z \in L^{2}\left([0, \infty) ; H^{s}\right)$. Moreover, $z(t, x)$ converges to $\bar{w}$ in $\mathscr{B}^{s-1}$-norm as $t \rightarrow \infty$. Consequently, $G(t, x)$ defined by (6.8) tends to $M$ in $\mathscr{B}^{s-1}$-norm as $t \rightarrow \infty$. (ii) If $w_{0}-\bar{w} \in H^{s} \cap L^{1}, s \geqq 1$, and $\stackrel{\circ}{E}_{s}=\left\|w_{0}-\bar{w}\right\|_{s}+\left\|w_{0}-\bar{w}\right\|_{L^{1}}$ is small, then

$$
\left\|\partial_{x}^{l}(z(t)-\bar{w})\right\|_{s-l},\left\|\partial_{x}^{l}(G(t)-M)\right\|_{s-l} \leqq C \dot{E}_{s}^{\circ}(1+t)^{-(1 / 2+l) / 2}
$$

for $t \in[0, \infty)$, where $0 \leqq l \leqq s$ and $C$ is a constant.

Next we shall show that the solution $F(t, x)$ of $(1.5),(1.6)$ constructed in Theorem 3.1 is well approximated by $G(t, x)$ for $t \rightarrow \infty$. We define $f(t, x)$ and $g(t, x)$ by (3.2) and (6.3), respectively. Then we get the formulas (6.1) and (6.4). It follows from (6.1) and (3.8) that for $0 \leqq j \leqq s$,

$$
\left\|(i \xi)^{j} \hat{f}(t, \xi)\right\|_{L^{2}\left(\left.\right|^{k} \mid \geqq \delta\right)} \leqq C e^{-c t}\left\|\partial_{x}^{j} f_{0}\right\|+C \int_{0}^{t} e^{-c(t-\tau)}\left\|\partial_{x}^{j} \Gamma_{M}(f, f)(\tau)\right\| d \tau .
$$

Here and in the sequel, $C$ and $c$ denote positive constants. Similarly, we have 
from (6.4),

$$
\left\|(i \xi)^{j} \hat{g}(t, \xi)\right\|_{L^{2}(\xi \xi \mid \geqq \delta)} \leqq C e^{-c t}\left\|\partial_{x}^{j} f_{0}\right\|+C\left(\int_{0}^{t} e^{-c(t-\tau)}\left\|\partial_{x}^{j} \Gamma_{M}(g, g)(\tau)\right\|^{2} d \tau\right)^{1 / 2} .
$$

For $|\xi|<\delta$, we use the expression (6.2). Subtracting (6.4) from (6.2) and using the expansions of $\mu_{j}(i \xi)$ and $\zeta_{j}(i \xi)$ (see Lemma 5.1), we obtain

$$
\begin{aligned}
|\hat{f}(t, \xi)-\hat{g}(t, \xi)| \leqq & C e^{-c t}\left|\hat{f}_{0}(\xi)\right|+C|\xi| e^{-c t \xi^{2}}\left|\hat{f}_{0}(\xi)\right| \\
& +C \int_{0}^{t} e^{-c(t-\tau)}\left|\hat{\Gamma}_{M}(f, f)(\tau, \xi)\right| d \tau \\
& +C \int_{0}^{t} \xi^{2} e^{-c(t-\tau) \xi^{2}}\left|\hat{\Gamma}_{M}(f, f)(\tau, \xi)\right| d \tau \\
& +C \int_{0}^{t}|\xi| e^{-c(t-\tau) \xi^{2}}\left|\hat{\Gamma}_{M}(f-g, f+g)(\tau, \xi)\right| d \tau, \quad|\xi|<\delta .
\end{aligned}
$$

Here we also used (5.11) and the fact that $\Gamma_{M}(f, f)$ is prependicular to $e_{j}, j=1, \ldots, d$. From (6.26) we get for $0 \leqq j \leqq s$,

$$
\begin{aligned}
& \left\|(i \xi)^{j}(\hat{f}(t, \xi)-\hat{g}(t, \xi))\right\|_{L^{2}(|\xi|<\delta)} \\
& \leqq \\
& \quad C e^{-c t}\left\|\partial_{x}^{j} f_{0}\right\|+C(1+t)^{-(3 / 2+j) / 2}\left\|f_{0}\right\|_{L^{1}} \\
& \quad+C \int_{0}^{t} e^{-c(t-\tau)}\left\|\partial_{x}^{j} \Gamma_{M}(f, f)(\tau)\right\| d \tau \\
& \quad+C \int_{0}^{t}(1+t-\tau)^{-(5 / 2+j-k) / 2}\left\|\partial_{x}^{k} \Gamma_{M}(f, f)(\tau)\right\|_{L^{1}} d \tau \\
& \quad+C \int_{0}^{t}(1+t-\tau)^{-\left(3 / 2+j-k^{\prime}\right) / 2}\left\|\partial_{x}^{k^{\prime}} \Gamma_{M}(f-g, f+g)(\tau)\right\|_{L^{1}} d \tau,
\end{aligned}
$$

where $0 \leqq k \leqq j+2$ and $0 \leqq k^{\prime} \leqq j+1$. Combining (6.24), (6.25) and (6.27), we have a desired approximation result.

Theorem 6.2. Assume Conditions 1 and 2. Suppose that $F_{0}-M \in H^{s} \cap L^{1}, s \geqq 1$, and $E_{s}=\left\|F_{0}-M\right\|_{s}+\left\|F_{0}-M\right\|_{L^{1}}$ is small. Let $F(t, x)$ be the solution of (1.5), (1.6) constructed in Theorem 3.1 and let $G(t, x)$ be the function in Proposition 6.1. Then we have

$$
\left\|\partial_{x}^{l}(F(t)-G(t))\right\|_{s-l} \leqq C E_{s}(1+t)^{-(3 / 2+l) / 2+\alpha}
$$

for $t \in[0, \infty)$, where $0 \leqq l \leqq s-1, C$ is a constant, and $\alpha>0$ is a small fixed constant.

Remark 6.1. A similar approximation result was proved in [12] (Theorem 3.9) for the one-dimensional Broadwell model of the Boltzmann equation.

Proof of Theorem 6.2. For $0 \leqq l \leqq s-1$, we define

$$
M_{l}(t)=\sup _{0 \leqq \tau \leqq t}(1+\tau)^{(3 / 2+l) / 2-\alpha}\left\|\partial_{x}^{l}(f(\tau)-g(\tau))\right\|_{s-l} .
$$


It suffices to show the inequality $M_{l}(t) \leqq C E_{s}$. First we have from (6.24), (6.25) and (6.27) with $0 \leqq j \leqq s$,

$$
\begin{aligned}
& \|f(t)-g(t)\|_{s} \leqq C E_{S}(1+t)^{-3 / 4}+C \int_{0}^{t} e^{-c(t-\tau)} . \\
& \left\|\Gamma_{M}(f, f)(\tau)\right\|_{s} d \tau+C\left(\int_{0}^{t} e^{-c(t-\tau)}\left\|\Gamma_{M}(g, g)(\tau)\right\|_{s}^{2} d \tau\right)^{1 / 2} \\
& \quad+C \int_{0}^{t / 2}(1+t-\tau)^{-5 / 4}\left\|\Gamma_{M}(f, f)(\tau)\right\|_{L^{1}} d \tau+C \int_{t / 2}^{t}(1+t-\tau)^{-3 / 4} . \\
& \left\|\partial_{\lambda} \Gamma_{M}(f, f)(\tau)\right\|_{L^{1}} d \tau+C \int_{0}^{t}(1+t-\tau)^{-3 / 4}\left\|\Gamma_{M}(f-g, f+g)(\tau)\right\|_{L^{1}} d \tau .
\end{aligned}
$$

Here we have taken $k$ and $k^{\prime}$ in (6.27) such that $k=0$ on $[0, t / 2], k=1$ on $[t / 2, t]$ and $k^{\prime}=0$ on $[0, t]$. We estimate each term on the right-hand side of $(6.30)$ by using (3.1) and (6.23) with $l=0,1$. By the same arguments as in the proof of (3.1), we know that the integrals except for the last one are all majorized by $C E_{s}^{2}(1+t)^{-3 / 4}$. For the last term, we have the bound

$$
C E_{s} M_{0}(t) \int_{0}^{t}(1+t-\tau)^{-3 / 4}(1+\tau)^{-1+\alpha} d \tau \leqq C E_{s} M_{0}(t)(1+t)^{-3 / 4+\alpha},
$$

where we used the definition of $M_{0}(t)$. Combining these estimates, we arrive at the inequality $M_{0}(t) \leqq C E_{s}+C E_{s} M_{0}(t)$, which gives $M_{0}(t) \leqq C E_{s}$ for small $E_{s}$. Thus the proof of (6.28) with $l=0$ is completed.

Next, from (6.24), (6.25) and (6.27) with $1 \leqq j \leqq s$, we obtain

$$
\left\|\partial_{x}(f(t)-g(t))\right\|_{s-1} \leqq C E_{s}(1+t)^{-5 / 4}+\cdots .
$$

Here we take $k$ and $k^{\prime}$ in (6.27) such that $k=k^{\prime}=0$ on $[0, t / 2]$, and $k=2, k^{\prime}=1$ on $[t / 2, t]$. We estimate each term on the right-hand side of $(6.31)$ by using $(3.1),(6.23)$ with $l=0,1,2$ and (6.28) with $l=0$. Similarly as in the above, we reach the inequality $M_{1}(t) \leqq C E_{s}+C E_{s} M_{1}(t)$, from which follows the estimate (6.28) with $l=1$ if $E_{s}$ is small. The estimates for higher derivatives are shown in the way and we omit the details.

\section{Approximation by Uniformly Parabolic System, II}

We shall give a modification of $G(t, x)$ in Proposition 6.1. Taking into account of $(6.8),(6.14)$ and $(6.15)$, we defined $G^{\prime}(t, x)$ by

$$
G^{\prime}(t, x)-M=\sum_{j=1}^{d}\left(z_{j}^{\prime}(t, x)-\bar{w}_{j}\right) \phi^{(j)},
$$

where $z^{\prime}(t, x)={ }^{t}\left(z_{1}^{\prime}, \ldots, z_{d}^{\prime}\right)(t, x)$ is a solution of the problem

$$
\begin{gathered}
z_{t}^{\prime}+\tilde{h}\left(z^{\prime}\right)_{x}=\tilde{D}(\bar{w}) z_{x x}^{\prime} \\
z^{\prime}(0, x)=w_{0}(x) \equiv{ }^{t}\left(\left\langle F_{0}(x), \psi^{(i)}\right\rangle\right)_{1 \leqq 1 \leqq d} .
\end{gathered}
$$

Here $\tilde{h}(z)$ and $\widetilde{D}(\bar{w})$ are defined by (4.9) and (6.16), respectively. Note that 
$z_{j}^{\prime}(t, x)=\left\langle G^{\prime}(t, x), \psi^{(j)}\right\rangle$, namely, $z_{j}^{\prime}(t, x)$ is the $j$-th moment of $G^{\prime}(t, x), j=1, \ldots, d$. The system (7.2) is semilinear and uniformly parabolic, and its linearized system around $z^{\prime}=\bar{w}$ agree with (6.18). Therefore we have the following

Proposition 7.1. Assume Conditions 1 and 2. (i) If $w_{0}-\bar{w} \in H^{s}, s \geqq 1$, and $\left\|w_{0}-\bar{w}\right\|_{s}$ is small, then the problem (7.2), (7.3) has a unique global solution $z^{\prime}(t, x)$ which converges to $\bar{w}$ in $\mathscr{B}^{s-1}$-norms as $t \rightarrow \infty$. Consequently, $G^{\prime}(t, x)$ defined by $(7.1)$ tends to $M$ in $\mathscr{B}^{s-1}$-norm as $t \rightarrow \infty$. (ii) If $w_{0}-\bar{w} \in H^{s} \cap L^{1}, s \geqq 1$, and $E_{s}=\left\|w_{0}-\bar{w}\right\|_{s}+$ $\left\|w_{0}-\bar{w}\right\|_{L^{1}}$ is small, then

$$
\left\|\partial_{x}^{l}\left(z^{\prime}(t)-\bar{w}\right)\right\|_{s-l}, \quad\left\|\partial_{x}^{l}\left(G^{\prime}(t)-M\right)\right\|_{s-l} \leqq C E_{s}^{\circ}(1+t)^{-(1 / 2+l) / 2}
$$

for $t \in[0, \infty]$, where $0 \leqq l \leqq s$ and $C$ is a constant.

We wish to show that $z^{\prime}(t, x)$ is well approximated by $z(t, x)$ in Proposition 6.1 for $t \rightarrow \infty$. For this purpose we prepare the following

Lemma 7.2. Let $\tilde{h}(z)$ and $k(z)$ be the functions in (4.9) and (6.16), respectively. Then we have

$$
|\tilde{h}(z)-k(z)|=O\left(|z-\bar{w}|^{3}\right) \quad \text { for } \quad|z-\bar{w}| \rightarrow 0 .
$$

Proof. Recall that the Euler equation (4.8) is equivalent to

$$
\left(\left\langle F^{(0)}, \psi^{(j)}\right\rangle\right)_{t}+\left(\left\langle V F^{(0)}, \psi^{(j)}\right\rangle\right)_{x}=0, \quad j=1, \ldots, d,
$$

where $F^{(0)}$ is determined by $(4.4)_{0}$ and $(4.2)_{0}$. We write

$$
F^{(0)}=M+\Lambda_{M}^{1 / 2} f \text {. }
$$

Substitution of (7.6) into (4.4) 0 yields

$$
L_{M} f=\Gamma_{M}(f, f) .
$$

Since $\Gamma_{M}(f, f) \in N\left(L_{M}\right)^{\perp}$ by Lemma 2.2, Eq. (7.7) is reduced to $f=P_{0} f+$ $L_{M}^{-1} \Gamma_{M}(f, f)$, where $P_{0}$ is the orthogonal projection onto $N\left(L_{M}\right)$, i.e., $P_{0} f=\sum_{j=1}^{d} f^{j} e_{j}$ with $f^{j}=\left\langle f, e_{j}\right\rangle, j=1, \ldots, d$. Therefore we get the formula

$$
f=\sum_{j=1}^{d} f^{j} e_{j}+\sum_{k, l=1}^{d} L_{M}^{-1} \Gamma_{M}\left(e_{k}, e_{l}\right) f^{k} f^{l}+R[f],
$$

where $R[f]=O\left(|f|^{3}\right)$ for $|f| \rightarrow 0$. By the definitions of $f$ and $e_{j}, j=1, \ldots, d$, we know that (7.5) is equivalent to

$$
\left(\left\langle f, e_{j}\right\rangle\right)_{t}+\left(\left\langle V f, e_{j}\right\rangle\right)_{x}=0, \quad j=1, \ldots, d .
$$

We substitute (7.8) into (7.9) and compute the resulting equation by using (5.6) and $\Gamma_{M}\left(e_{k}, e_{l}\right) \in N\left(L_{M}\right)^{\perp}$. We then obtain

$$
f_{t}^{j}+\lambda_{j}(\bar{w}) f_{x}^{j}+\sum_{k, l=1}^{d} q_{k l}^{j}(\bar{w})\left(f^{k} f^{l}\right)_{x}+R^{j}[f]_{x}=0, \quad j=1, \ldots, d,
$$

where the coefficients $q_{k l}^{j}(\bar{w})$ are in (6.11), and $R^{j}[f]=\left\langle V R[f], e_{j}\right\rangle, j=1, \ldots, d$. We rewrite (7.10) into the equation of the moments $w_{j}, j=1, \ldots, d$, of $F^{(0)}$. Similarly as in 
the proof of (6.14), we arrive at

$$
w_{t}+(k(w)+r(w))_{x}=0
$$

where $w={ }^{t}\left(w_{1}, \ldots, w_{d}\right), k(w)$ is in (6.16), and

$$
r(w)=\sum_{j=1}^{d} R^{j}[f] c_{j}(\bar{w})^{1 / 2} \tilde{r}_{j}(\bar{w}) .
$$

Notice that the right-hand side of (7.12) is a function of $w$ because $F^{(0)}$ and hence $f$ is a function of $w$. Also, note that $r(w)=O\left(|f|^{3}\right)=O\left(|w-\bar{w}|^{3}\right)$ for $|w-\bar{w}| \rightarrow 0$. Equation (7.11) must agree with (4.8). Therefore we get $\widetilde{h}(w)=k(w)+r(w)$. Thus the proof of Lemma 7.2 is completed.

By virtue of Lemma 7.2 we have

Proposition 7.3. Asuume Conditions 1 and 2. Suppose that $w_{0}-\bar{w} \in H^{s} \cap L^{1}, s \geqq 1$, and $\dot{E}_{s}^{\circ}=\left\|w_{0}-\bar{w}\right\|_{s}+\left\|w_{0}-\bar{w}\right\|_{L^{1}}$ is small. Let $G(t, x)$ and $G^{\prime}(t, x)$ be the functions in Propositions 6.1 and 7.1 , respectively. Then we have

$$
\left\|\partial_{x}^{l}\left(G^{\prime}(t)-G(t)\right)\right\|_{s-l} \leqq C \stackrel{\circ}{2}_{s}^{2}(1+t)^{-(3 / 2+l) / 2+\alpha}
$$

for $t \in[0, \infty)$, where $0 \leqq l \leqq s, C$ is a constant, and $\alpha>0$ is a small fixed constant.

A combination of Theorem 6.2 and Proposition 7.3 gives the following

Corollary 7.4. Assume the same conditions of Theorem 6.2. Let $F(t, x)$ be the solution of (1.5), (1.6) constructed in Theorem 3.1 and let $G^{\prime}(t, x)$ be the function in Proposition 7.1. Then we have

$$
\left\|\partial_{x}^{l}\left(F(t)-G^{\prime}(t)\right)\right\|_{s-l} \leqq C E_{\mathrm{s}}(1+t)^{-(3 / 2+l) / 2+\alpha}
$$

for $t \in[0, \infty)$, where $0 \leqq l \leqq s-1, C$ is a constant, and $\alpha>0$ is a small fixed constant. Proof of Proposition 7.3. It suffices to show the estimate

$$
\left\|\partial_{x}^{l}\left(z^{\prime}(t)-z(t)\right)\right\|_{s-l} \leqq C \dot{E}_{s}^{2}(1+t)^{-(3 / 2+l) / 2+\alpha}
$$

for $t \in[0, \infty)$, where $0 \leqq l \leqq s$. Here $z(t, x)$ and $z^{\prime}(t, x)$ are the solutions of the problems $(6.14),(6.15)$ and $(7.2),(7.3)$, respectively. Using the semigroup $e^{t S}$ of $(6.18)$, we get

$$
z^{\prime}(t)-z(t)=-\int_{0}^{t} e^{(t-\tau) S} r\left(z^{\prime}\right)_{x}(\tau) d \tau-\int_{0}^{t} e^{(t-\tau) S}\left(q\left(z^{\prime}\right)-q(z)\right)_{x}(\tau) d \tau,
$$

where $r(z)$ and $q(z)$ are given by (7.12) and (6.17), respectively. Put

$$
M_{l}(t)=\sup _{0 \leqq t \leqq t}(1+\tau)^{(3 / 2+l) / 2-\alpha}\left\|\partial_{x}^{l}\left(z^{\prime}(t)-z(t)\right)\right\|_{s-l}
$$

for $0 \leqq l \leqq s$. We apply $\partial_{x}^{j}, 0 \leqq j \leqq s$, to (7.16) and estimate each equation by using (6.21) and (6.22). Summing up for $0 \leqq j \leqq s$, we obtain

$$
\begin{gathered}
\left\|z^{\prime}(t)-z(t)\right\|_{s} \leqq C\left(\int_{0}^{t} e^{-c(t-\tau)}\left\|r\left(z^{\prime}\right)(\tau)\right\|_{s}^{2} d \tau\right)^{1 / 2} \\
+C\left(\int_{0}^{t} e^{-c(t-\tau)}\left\|\left(q\left(z^{\prime}\right)-q(z)\right)(\tau)\right\|_{s}^{2} d \tau\right)^{1 / 2}
\end{gathered}
$$




$$
\begin{aligned}
& +C \int_{0}^{t}(1+t-\tau)^{-3 / 4}\left\|r\left(z^{\prime}\right)(\tau)\right\|_{L^{1}} d \tau \\
& +C \int_{0}^{t}(1+t-\tau)^{-3 / 4}\left\|\left(q\left(z^{\prime}\right)-q(z)\right)(\tau)\right\|_{L^{1}} d \tau .
\end{aligned}
$$

We estimate each term on the right-hand side of (7.18) by using (6.23) and (7.4) with $l=0,1$. The first and the second terms are estimated respectively by

$$
\begin{aligned}
& C \dot{E}_{s}^{3}\left(\int_{0}^{t} e^{-c(t-\tau)}(1+\tau)^{-5 / 2} d \tau\right)^{1 / 2} \leqq C E_{s}^{3}(1+t)^{-5 / 4}, \\
& C \dot{E}_{s}^{2}\left(\int_{0}^{t} e^{-c(t-\tau)}(1+\tau)^{-3 / 2} d \tau\right)^{1 / 2} \leqq C \dot{E}_{s}^{2}(1+t)^{-3 / 4} .
\end{aligned}
$$

For any fixed $\gamma>0$, the third term has the bound

$$
C E_{s}^{0} \int_{0}^{t}(1+t-\tau)^{-3 / 4}(1+\tau)^{-1} d \tau \leqq C E_{s}^{\circ}(1+t)^{-3 / 4+\gamma} .
$$

Similarly, the last term is majorized by

$$
C E_{s}^{\circ} M_{0}(t) \int_{0}^{t}(1+t-\tau)^{-3 / 4}(1+\tau)^{-1+\alpha} d \tau \leqq C E_{s}^{\circ} M_{0}(t)(1+t)^{-3 / 4+\alpha},
$$

where we used the definition of $M_{0}(t)$. Substituting these estimates into (7.18) and choosing $\gamma=\alpha$, we obtain the inequality $M_{0}(t) \leqq C E_{s}^{2}+C E_{s}^{\circ} M_{0}(t)$, which gives $M_{0}(t) \leqq C E_{s}^{2}$ if $E_{s}$ is small. Thus (7.15) with $l=0$ is proved. The estimates for the derivatives are shown in the same way and we omit the details.

\section{Large-time Behavior of Solutions}

An asymptotic solution of the semilinear uniformly parabolic system (7.2) was constructed in [14] by employing the technique of Liu [16]. We first review its construction. We determine $\delta_{j}(\bar{w}), j=1, \ldots, d$, by

$$
\int_{-\infty}^{\infty}\left(w_{0}(x)-\bar{w}\right) d x=\sum_{j=1}^{d} \delta_{j}(\bar{w}) \tilde{r}_{j}(\bar{w})
$$

where $w_{0}(x)={ }^{t}\left(\left\langle F_{0}(x), \psi^{(j)}\right\rangle\right)_{1 \leqq j \leqq d}$ and $\tilde{r}_{j}(w), j=1, \ldots, d$, are the right eigenvectors of $\widetilde{A}(w)$. Put $\delta(\bar{w})={ }^{t}\left(\delta_{1}(\bar{w}), \ldots, \delta_{d}(\bar{w})\right)$ and assume that $|\delta(\bar{w})| \neq 0$. We denote by $R_{j}(\bar{w})$ the integral curve of the vector $\tilde{r}_{j}(w)$ through the point $w=\bar{w}$. When $j$-th characteristic field is genuinely nonlinear, we define the nonlinear diffusion wave $\bar{w}^{j}(t, x)$ by

$$
\begin{gathered}
\bar{w}^{j}(t, x) \in R_{j}(\bar{w}), \\
\lambda_{j}\left(\bar{w}^{j}(t, x)\right)-\lambda_{j}(\bar{w})=y\left(t+1, x-\lambda_{j}(\bar{w})(t+1) ; \kappa_{j}(\bar{w}), \delta_{j}(\bar{w})\right),
\end{gathered}
$$

where

$$
y(t, x ; \kappa, \delta)=\sqrt{\kappa} t^{-1 / 2} \frac{\left(e^{\delta / 2 \kappa}-1\right) e^{-\eta^{2}}}{\sqrt{\pi}+\left(e^{\delta / 2 \kappa}-1\right) \int_{\eta}^{\infty} e^{-\xi^{2}} d \xi}, \quad \eta=x / \sqrt{4 \kappa t} .
$$


Note that $y=y(t, x ; \kappa, \delta)$ is the self-similar solution of the Burgers equation $y_{t}+y y_{x}=\kappa y_{x x}$ and satisfies

$$
\int_{-\infty}^{\infty} y(t, x ; \kappa, \delta) d x=\delta \quad \text { for } \quad t>0
$$

In $(8.2)_{1}, \lambda_{j}(w)$ is the eigenvalue of $\widetilde{A}(w)$ and $\kappa_{j}(\bar{w})$ is the coefficient determined by $(5.3)_{2}$. Since $\left\langle\nabla_{w} \lambda_{j}(w), \tilde{r}_{j}(w)\right\rangle=1$ by $(4.12), \bar{w}^{j}(t, x)$ is uniquely determined by $(8.2)_{1}$, provided that $\delta_{j}(\bar{w})$ is small. When $j$-th characteristic field is linearly degenerate, we choose a smooth function $s_{j}=s_{j}(w)$ such that $s_{j}(\bar{w})=\lambda_{j}(\bar{w})$ and $\left\langle\nabla_{w} s_{j}(w), \tilde{r}_{j}(w)\right\rangle=1$, and then define the linear diffusion wave $\bar{w}^{j}(t, x)$ by

$$
\begin{gathered}
\bar{w}^{j}(t, x) \in R_{j}(\bar{w}), \\
s_{j}\left(\bar{w}^{j}(t, x)\right)-\lambda_{j}(\bar{w})=y\left(t+1, x-\lambda_{j}(\bar{w})(t+1) ; \kappa_{j}(\bar{w}), \delta_{j}(\bar{w})\right),
\end{gathered}
$$

where

$$
y(t, x ; \kappa, \delta)=\delta(4 \pi \kappa t)^{-1 / 2} e^{-\eta^{2}}, \quad \eta=x / \sqrt{4 \kappa t} .
$$

Note that $y=y(t, x ; \kappa, \delta)$ is the self-similar solution of the linear heat equation $y_{t}=\kappa y_{x x}$ and satisfies (8.4). Note also that $\bar{w}^{j}(t, x)$ is uniquely determined by $(8.2)_{2}$ for small $\delta_{j}(\bar{w})$.

Now, we define $\bar{w}(t, x)$, the superposition of the diffusion waves, by

$$
\bar{w}(t, x)-\bar{w}=\sum_{j=1}^{d}\left(\bar{w}^{j}(t, x)-\bar{w}\right) .
$$

Then, by Theorem 8.2 of $[14], \bar{w}(t, x)$ is an asymptotic solution for $t \rightarrow \infty$ of the problems (7.2), (7.3). Indeed, if $w_{0}-\bar{w} \in H^{s} \cap L_{\beta}^{1}, s \geqq 1, \beta \in[0,1]$, and $E_{s}^{\circ}=$ $\left\|w_{0}-\bar{w}\right\|_{s}+\left\|w_{0}-\bar{w}\right\|_{L^{1}}$ is small, then we have

$$
\left\|\partial_{x}^{l}\left(z^{\prime}(t)-\bar{w}(t)\right)\right\|_{s-l} \leqq C E_{s, \beta}^{\circ}(1+t)^{-(1+l) / 2+\alpha}
$$

for $t \in[0, \infty)$, where $0 \leqq l \leqq s$ and $C$ is a constant. Here $\alpha=(1 / 2-\beta) / 2$ if $\beta \in[0,1 / 2)$, and $\alpha>0$ is a small fixed constant if $\beta \in[1 / 2,1] . E_{s, \beta}^{\circ}$ is given by

$$
\stackrel{\circ}{E}, \beta_{s,}=\left\{\begin{array}{lll}
\left\|w_{0}-\bar{w}\right\|_{s}+\left\|w_{0}-\bar{w}\right\|_{L_{\beta}^{1}} & \text { if } & \beta \in[0,1 / 2), \\
\left\|w_{0}-\bar{w}\right\|_{s}+\left\|w_{0}-\bar{w}\right\|_{L_{1 / 2}^{1}} & \text { if } & \beta \in[1 / 2,1] .
\end{array}\right.
$$

As a consequence of (8.6), the function $G^{\prime}(t, x)$ in Proposition 7.1 is approximated for $t \rightarrow \infty$ by the following function $\bar{F}(t, x)$ :

$$
\bar{F}(t, x)-M=\sum_{j=1}^{d}\left(\bar{w}_{j}(t, x)-\bar{w}_{j}\right) \phi^{(j)},
$$

where $\bar{w}_{j}(t, x)$ is $j$-th component of $\bar{w}(t, x)$ in (8.5). Note that $\bar{w}_{j}(t, x)=\left\langle\bar{F}(t, x), \psi^{(j)}\right\rangle$, namely, $\bar{w}_{j}(t, x)$ is the $j$-th moment of $\bar{F}(t, x), j=1, \ldots, d$. These considerations are summarized as follows.

Theorem 8.1. Assume Conditions 1 and 2. Let $s \geqq 1$ and $\beta \in[0,1]$. Suppose that $w_{0}$ $-\bar{w} \in H^{s} \cap L_{\beta}^{1}$ and $E_{s}^{\circ}=\left\|w_{0}-\bar{w}\right\|_{s}+\left\|w_{0}-\bar{w}\right\|_{L^{1}}$ is small. Let $G^{\prime}(t, x)$ be the function in Proposition 7.1 and let $\bar{F}(t, x)$ be the function defined by (8.8) in terms of the 
superposition of the diffusion waves. Then we have

$$
\left\|\partial_{x}^{l}\left(G^{\prime}(t)-\bar{F}(t)\right)\right\|_{s-1} \leqq C E_{s, \beta}^{\circ}(1+t)^{-(1+l) / 2+\alpha}
$$

for $r \in[0, \infty)$, where $0 \leqq l \leqq s, C$ is a constant, and $\dot{E}_{s, \beta}$ is given by (8.7). Here $\alpha=$ $(1 / 2-\beta) / 2$ if $\beta \in[0,1 / 2)$, and $\alpha>0$ is a small fixed constant if $\beta \in[1 / 2,1]$.

A combination of Corollary 7.4 and Theorem 8.1 gives the main theorem of this paper.

Theorem. 8.2. Assume Conditions 1 and 2. Let $s \geqq 1$ and $\beta \in[0,1]$. Suppose that $F_{0}-M \in H^{s} \cap L^{1}$ and $w_{0}-\bar{w} \in L_{\beta}^{1}$ and that $E_{s}=\left\|F_{0}-M\right\|_{s}+\left\|F_{0}-M\right\|_{L^{1}}$ is small. Let $F(t, x)$ be the solution of (1.5), (1.6) constructed in Theorem 3.1 and let $\bar{F}(t, x)$ be the function in Theorem 8.1. Then we have

$$
\left\|\partial_{x}^{l}(F(t)-\bar{F}(t))\right\|_{s-l} \leqq C E_{s, \beta}(1+t)^{-(1+l) / 2+\alpha}
$$

for $t \in[0, \infty)$, where $0 \leqq l \leqq s-1, C$ is a constant, $E_{s, \beta}=\max \left\{E_{s}, \dot{E}_{s, \beta}\right\}$, and $\alpha>0$ is $a$ constant determined in Theorem 8.1.

Remark. 8.1. When $|\delta(\bar{w})| \neq 0$, we conclude from Lemma 7.1 of [14] that for large $t$,

$$
\left\|\partial_{x}^{l}(\bar{F}(t)-M)\right\| \geqq c|\delta(\bar{w})|(1+t)^{-(1 / 2+l) / 2},
$$

where $l \geqq 0$ and $c$ is a positive constant. Therefore, the estimates (6.28), (7.13), (7.14), (8.9) and (8.10) give meaningful asymptotic relations for $t \rightarrow \infty$. In particular, $\bar{F}(t, x)$ constructed by the superposition of the diffusion waves is an asymptotic solution for $t \rightarrow \infty$ of the discrete Boltzmann equation (1.5).

\section{The Broadwell Model in One Space Dimension}

As an application of our general result, we treat here the simplest model proposed by Broadwell [2]. We consider the following six velocities.

$$
\begin{aligned}
\mathbb{U}_{1} & =(v, 0,0), \quad \mathbb{U}_{2}=(0, v, 0), \quad \mathbb{U}_{3}=(0,0, v), \\
\mathbb{U}_{p+3} & =-\mathbb{U}_{p}, \quad p=1,2,3,
\end{aligned}
$$

where $v$ is a positive constant. We denote by $N_{p}=N_{p}(t, \mathbb{X})(p=1, \ldots, 6)$ the mass density of gas particles with the velocity $\mathbb{U}_{p}$ at time $t \geqq 0$ and position $\mathbb{X}=$ $(x, y, z) \in \mathbb{R}^{3}$. We assume that $N_{p}(t, \mathbb{X}), p=1, \ldots, 6$, do not depend on $y$ and $z$, i.e., $\quad N_{p}(t, \mathbb{X})=N_{p}(t, x)$, and that $N_{2}(t, x)=N_{3}(t, x)=N_{5}(t, x)=N_{6}(t, x)$. Put $F_{1}(t, x)=N_{1}(t, x), F_{2}(t, x)=N_{2}(t, x)$ and $F_{3}(t, x)=N_{4}(t, x)$. The original Broadwell model in three space dimensions is then reduced to $([2])$

$$
F_{t}+V F_{x}=Q(F, F)
$$

where $F={ }^{t}\left(F_{1}, F_{2}, F_{3}\right), V=\operatorname{diag}(v, 0,-v)$ and

$$
Q(F, G)=\frac{\bar{\sigma}}{2}\left\{2 F_{2} G_{2}-\left(F_{1} G_{3}+F_{3} G_{1}\right)\right\}\left(\begin{array}{c}
1 \\
-1 / 2 \\
1
\end{array}\right)
$$

with a positive constants $\bar{\sigma}$. The component of $Q(F, G)$ in $(9.2)$ are of the same form as 
in (1.4) if we put

$$
\begin{gathered}
\alpha_{1}=\alpha_{3}=1, \quad \alpha_{2}=4 \\
A_{22}^{13}=A_{22}^{31}=A_{13}^{22}=A_{31}^{22}=\bar{\sigma} \text { and } A_{k l}^{i j}=0 \text { otherwise. }
\end{gathered}
$$

Consequently, (1.2) and (1.3) are satisfied.

The space $\mathscr{M}$ of summational invariants consists of vectors $\psi={ }^{t}\left(\psi_{1}, \psi_{2}, \psi_{3}\right)$ satisfying $\psi_{1}+\psi_{3}-\psi_{2} / 2=0$. Therefore, putting

$$
\psi^{(1)}={ }^{t}(1,4,1), \quad \psi^{(2)}={ }^{t}(v, 0,-v),
$$

we see that $\left\{\psi^{(1)}, \psi^{(2)}\right\}$ is a basis of $\mathscr{M}$. Note that $\mathscr{M}^{\perp}$ is spanned by $\phi^{(3)}=$ ${ }^{t}(1,-1 / 2,1)$. We denote the moments of $F={ }^{t}\left(F_{1}, F_{2}, F_{3}\right)$ by $\rho$ and $m$ :

$$
\rho=\left\langle F, \psi^{(1)}\right\rangle=F_{1}+4 F_{2}+F_{3}, \quad m=\left\langle F, \psi^{(2)}\right\rangle=v\left(F_{1}-F_{3}\right) .
$$

Let $F={ }^{t}\left(F_{1}, F_{2}, F_{3}\right)$ be a Maxwellian with positive components, namely $F_{1}, F_{2}$, $F_{3}>0$ and $F_{1} F_{3}-F_{2}^{2}=0$. By a simple calculation, we know that $F$ is expressed in terms of its moments $\rho$ and $m$ as follows (see [6]):

$$
F=\frac{\rho}{4 v^{2}}\left(\begin{array}{c}
2(\sigma(u)+v u) \\
v^{2}-\sigma(u) \\
2(\sigma(u)-v u)
\end{array}\right), \quad u=m / \rho .
$$

where $\rho>0,|u|<v$, and

$$
\sigma(u)=\left(v^{2} / 3\right)\left(2 \sigma_{0}(u)-1\right), \quad \sigma_{0}(u)=\left(1+3 u^{2} / v^{2}\right)^{1 / 2} .
$$

Note that $v|u|<\sigma(u)<v^{2}$ for $|u|<v$. By a straightforward calculation, using (9.5), we obtain the Euler equation for (9.1) (see for example, [6 or 12]):

$$
\rho_{t}+m_{x}=0, \quad m_{t}+(\rho \sigma(m / \rho))_{x}=0 .
$$

This is rewritten in the vector form (4.8) with the coefficient matrix $\tilde{A}$ :

$$
\tilde{A}=\left(\begin{array}{cc}
0 & 1 \\
\sigma(u)-u \sigma^{\prime}(u) & \sigma^{\prime}(u)
\end{array}\right), \quad u=m / \rho,
$$

where $\sigma^{\prime}(u)=d \sigma(u) / d u$.

Lemma 9.1. The one-dimensional Broadwell model (9.1) satisfies Conditions 1 and 2. In particular, the Euler equation (9.7) is strictly hyperbolic and both the charateristic fields are genuinely nonlinear in the region $\{\rho>0,|u|<v\}$.

Proof. Let $\psi \in \mathscr{M}$ and $\lambda \psi=V \psi$ for $\lambda \in \mathbb{R}$. Since $\mathscr{M}$ is spanned by $\psi^{(1)}$ and $\psi^{(2)}$, we may write $\psi=c_{1} \psi^{(1)}+c_{2} \psi^{(2)}$, where $c_{1}, c_{2} \in \mathbb{R}$. We substitute this expression into $\lambda \psi=V \psi$ to get $c_{1}(\lambda-V) \psi^{(1)}+c_{2}(\lambda-V) \psi^{(2)}=0$. A simple calculation shows that the vectors

$$
\begin{aligned}
& (\lambda-V) \psi^{(1)}={ }^{t}(\lambda-v, 4 \lambda, \lambda+v), \\
& (\lambda-V) \psi^{(2)}=v^{t}(\lambda-v, 0,-(\lambda+v))
\end{aligned}
$$

are linearly independent for any $\lambda \in \mathbb{R}$. This implies $c_{1}=c_{2}=0$ and hence $\psi=0$. 
Thus Condition 1 has been checked. We remark that in the previous paper [13] we verified the equivalent condition $(3.6)_{4}$.

Condition 2 had been checked in [5]. We give the outline of the proof. The eigenvalues $\lambda_{j}$ of $\tilde{A}$ are given explicitly by

$$
\begin{aligned}
& \lambda_{1}=\hat{\lambda}_{1}(u) \equiv\left(u-\sigma(u)^{1 / 2}\right) / \sigma_{0}(u), \\
& \lambda_{2}=\hat{\lambda}_{2}(u) \equiv\left(u+\sigma(u)^{1 / 2}\right) / \sigma_{0}(u), \quad u=m / \rho .
\end{aligned}
$$

A straightforward calculation shows that for $|u|<v$,

$$
\begin{gathered}
\hat{\lambda}_{j}^{\prime}(u)=d \lambda_{j}(u) / d u>0, \quad j=1,2, \\
-v<\hat{\lambda}_{1}(u)<\min \{u, 0\}<\max \{u, 0\}<\hat{\lambda}_{2}(u)<v .
\end{gathered}
$$

Therefore the Euler equation (9.7) is strictly hyperbolic. The right eigenvectors $\tilde{r}_{j}$ of $\tilde{A}$ for $\lambda_{j}$ are then given by

$$
\tilde{r}_{j}=a_{j}{ }^{t}\left(1, \lambda_{j}\right), \quad j=1,2,
$$

where $a_{j} \neq 0$. By a simple calculation, we see that $\left\langle\nabla \lambda_{j}, \tilde{r}_{j}\right\rangle=a_{j} \hat{\lambda}_{j}^{\prime}(u)\left(\hat{\lambda}_{j}(u)-u\right) / \rho \neq 0$ by $(9.10), j=1,2$, where $\nabla$ denotes the gradient with respect to $(\rho, m)$. This means that both the characteristic fields are genuinely nonlinear. The coefficients $a_{j}$ in (9.11) are determined by the normalization $\left\langle\nabla \lambda_{j}, \tilde{r}_{j}\right\rangle=1$ :

$$
a_{j}=\rho / \hat{\lambda}_{j}^{\prime}(u)\left(\hat{\lambda}_{j}(u)-u\right), \quad u=m / \rho, \quad j=1,2 .
$$

This completes the proof of Lemma 9.1.

By Lemma 9.1, we can apply all the results in the preceding sections to the onedimensional Broadwell model (9.1). In what follows we shall give the concrete form of the asymptotic solution of (9.1). First we determine the vectors $\phi^{(1)}$ and $\phi^{(2)}$ in (2.14), (2.15), which are used in the formula (8.8). Let $M={ }^{t}\left(M_{1}, M_{2}, M_{3}\right)$ be an absolute Maxwellian with positive components. We can write

$$
M=M_{2}{ }^{t}\left(a, 1, a^{-1}\right), \quad M_{2}>0, \quad a=M_{1} / M_{2}>0 .
$$

The matrix $\Lambda_{M}$ in (2.10) is then given by $\Lambda_{M}=M_{2} \operatorname{diag}\left(a, 1 / 4, a^{-1}\right)$. We compute $\phi^{(1)}$ and $\phi^{(2)}$ by the formula $\phi^{(j)}=\sum_{k=1}^{2} c_{j k} \Lambda_{M} \psi^{(k)}, j=1,2$, where $c_{j k}$ are the elements of the inverse matrix of $\left(\left\langle\Lambda_{M} \psi^{(j)}, \psi^{(k)}\right\rangle\right)_{1 \leqq 1, k \leqq 2}$. By a straightforward calculation, we get

$$
\phi^{(1)}=\frac{1}{4 b}\left(\begin{array}{c}
2 \\
a+a^{-1} \\
2
\end{array}\right), \quad \phi^{(2)}=\frac{1}{4 b}\left(\begin{array}{c}
2(1+2 a) \\
-\left(a-a^{-1}\right) \\
-2\left(1+2 a^{-1}\right)
\end{array}\right)
$$

where $b=a+1+a^{-1}$.

Next, we determine the diffusion coefficients $\kappa_{j}$ in $(5.3)_{2}$, which are used in $(8.2)_{1}$. Let $L_{M}$ be the matrix defined by (2.11). We have the expression

$$
L_{M}=\bar{\sigma} M_{2}\left(\begin{array}{ccc}
a^{-1} & -a^{-1 / 2} & 1 \\
-a^{-1 / 2} & 1 & -a^{1 / 2} \\
1 & -a^{1 / 2} & a
\end{array}\right)
$$


We compute the characteristic polynomial of the matrix $B(i \xi)=-\left(L_{M}+i \xi V\right), \xi \in \mathbb{R}$. Put $L_{M}=\bar{\sigma} M_{2} \hat{L}_{M}$ and $V=v \hat{V}$. We have $B(i \zeta)=\bar{\sigma} M_{2} \hat{B}(\eta)$ with $\eta=i \bar{\xi} v / \bar{\sigma} M_{2}$, where $\hat{B}(\eta)=-\left(\hat{L}_{M}+\eta \hat{V}\right)$. A straightforward calculation gives (cf. [10])

$$
\operatorname{det}(\hat{\mu} I-\hat{B}(\eta))=\hat{\mu}^{3}+b \hat{\mu}^{2}+\left(c \eta-\eta^{2}\right) \hat{\mu}-\eta^{2},
$$

where $b=a+1+a^{-1}$ and $c=a-a^{-1}$. We denote by $\hat{\mu}_{j}(\eta), j=1,2$, the eigenvalues of $\hat{B}(\eta)$ satisfying $\hat{\mu}_{j}(\eta) \rightarrow 0$ for $\eta \rightarrow 0$. We determine the coefficients in the expansions $\hat{\mu}_{j}(\eta)=\sum_{n=1}^{\infty} \eta^{n} \hat{\mu}_{j}^{(n)}, j=1,2$, by using (9.16) (see [10]). Since the eigenvalues $\mu_{j}(i \xi)$ of

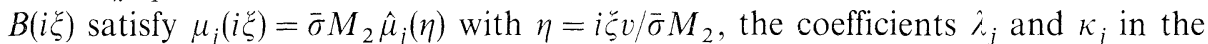
expansions of $\mu_{j}(i \xi)$ (see (5.7) and (5.8)) are calculated by the formulas $\lambda_{j}=-v \hat{\mu}_{j}^{(1)}$ and $\kappa_{j}=v^{2} \hat{\mu}_{j}^{(2)} / \bar{\sigma} M_{2}$. Therefore we obtain

$$
\begin{array}{ll}
\lambda_{1}=v\left(c-D^{1 / 2}\right) / 2 b, & \lambda_{2}=v\left(c+D^{1 / 2}\right) / 2 b, \\
\kappa_{1}=-\lambda_{1}\left(v^{2}-\lambda_{1}^{2}\right) / v \bar{\sigma} M_{2} D^{1 / 2}, & \kappa_{2}=\lambda_{2}\left(v^{2}-\lambda_{2}^{2}\right) / v \bar{\sigma} M_{2} D^{1 / 2},
\end{array}
$$

where $D=c^{2}+4 b, b=a+1+a^{-1}$ and $c=a-a^{-1}$. We note that $\lambda_{j}$ in (9.17) agree with $\hat{i}_{j}(\bar{u}), \bar{u}=\bar{m} / \bar{\rho}$, in (9.9), where $\bar{\rho}$ and $\bar{m}$ are the moments of $M$ :

$$
\bar{\rho}=\left\langle M, \psi^{(1)}\right\rangle=M_{2}\left(a+4+a^{-1}\right), \quad \bar{m}=\left\langle M, \psi^{(2)}\right\rangle=v M_{2}\left(a-a^{-1}\right) .
$$

Note also that $\kappa_{j}$ in (9.17) satisfy $\kappa_{j}>0$ by $(9.10)$.

Finally, we determine the integral curve $R_{j}$ of the right eigenvector $\tilde{r}_{j}$ through the point $(\bar{\rho}, \bar{m})$. Let $z_{j}(\rho, m)$ be the $j$-th Riemann invariant, i.e., $\left\langle\nabla z_{j}, \tilde{r}_{j}\right\rangle=0$ for all $(\rho, m)$, where $\nabla$ denotes the gradient with respect to $(\rho, m)$. By a straightforward calculation, using (9.11), we obtain

$$
z_{j}(\rho, m)=\rho^{2} \exp \left(-2 \int_{0}^{u}\left(\hat{\lambda}_{j}(\eta)-\eta\right)^{-1} d \eta\right), \quad u=m / \rho, \quad j=1,2 .
$$

The integral curve $R_{j}$ is then expressed by the equation $z_{j}(\rho, m)=z_{j}(\bar{\rho}, \bar{m})$, namely,

$$
\begin{gathered}
\rho=\bar{\rho} \exp \left(\int_{\bar{u}}^{u}\left(\hat{\lambda}_{,},(\eta)-\eta\right)^{-1} d \eta\right), \quad \bar{u}=\bar{m} / \bar{\rho}, \\
m=\rho u, \quad j=1,2 .
\end{gathered}
$$

Now we define the asymptotic solution $\bar{F}(t, x)$ of $(9.1)$. Let $F_{0}(x)$ be the initial data and define its moments $\rho_{0}(x)$ and $m_{0}(x)$ by

$$
\rho_{0}(x)=\left\langle F_{0}(x), \psi^{(1)}\right\rangle, \quad m_{0}(x)=\left\langle F_{0}(x), \psi^{(2)}\right\rangle .
$$

We determine $\delta_{j}, j=1,2$, by

$$
\int_{-\infty}^{\infty}{ }^{t}\left(\rho_{0}(x)-\bar{\rho}, m_{0}(x)-\bar{m}\right) d x=\sum_{j=1}^{2} \delta_{j} \tilde{r}_{j}
$$

where $\tilde{r}_{j}$ are given by $(9.11),(9.12)$ and evaluated at the constant state $(\bar{\rho}, \bar{m})$. Since the integral curves $R$, are given by (9.20), the nonlinear diffusion waves $\left(\bar{\rho}^{j}, \bar{m}^{j}\right)(t, x)$ are 
determined by the formulas

$$
\begin{aligned}
\bar{\rho}^{j}(t, x) & =\bar{\rho} \exp \left(\int_{\bar{u}}^{\bar{u}^{j}(t, x)}\left(\hat{\lambda}_{j}(\eta)-\eta\right)^{-1} d \eta\right), \quad \bar{u}=\bar{m} / \bar{\rho}, \\
\bar{m}^{j}(t, x) & =\bar{\rho}^{j}(t, x) \bar{u}^{j}(t, x), \\
\hat{\lambda}_{j}\left(\bar{u}^{j}(t, x)\right)-\hat{\lambda}_{j}(\bar{u}) & =y\left(t+1, x-\hat{\lambda}_{j}(\bar{u})(t+1) ; \kappa_{j}, \delta_{j}\right), \quad j=1,2,
\end{aligned}
$$

where $\kappa_{j}$ and $\delta_{j}$ are given by (9.17) and (9.22), respectively, and $y(t, x ; \kappa, \delta)$ by $(8.3)_{1}$. We denote the superposition of the nonlinear diffusion waves by $(\bar{\rho}, \bar{m})(t, x)$ :

$$
\begin{aligned}
\bar{\rho}(t, x)-\bar{\rho} & =\sum_{j=1}^{2}\left(\bar{\rho}^{j}(t, x)-\bar{\rho}\right), \\
\bar{m}(t, x)-\bar{m} & =\sum_{j=1}^{2}\left(\bar{m}^{j}(t, x)-\bar{m}\right) .
\end{aligned}
$$

The asymptotic solution $\bar{F}(t, x)$ is then given by

$$
\bar{F}(t, x)-M=(\bar{\rho}(t, x)-\bar{\rho}) \phi^{(1)}+(\bar{m}(t, x)-\bar{m}) \phi^{(2)},
$$

where $\phi^{(1)}$ and $\phi^{(2)}$ are the vectors in (9.14).

From Lemma 9.1 and Theorem 8.2 we have

Theorem 9.2. Let $M$ be an absolute Maxwellian with positive components and let $(\bar{\rho}, \bar{m})$ be its moments. Assume that $F_{0}-M \in H^{s} \cap L^{1}$ and $\left(\rho_{0}-\bar{\rho}, m_{0}-\bar{m}\right) \in L_{\beta}^{1}$ for $s \geqq 1$ and $\beta \in[0,1]$, where $\left(\rho_{0}, m_{0}\right)(x)$ are the moments of $F_{0}(x)$. If $E_{s}=\left\|F_{0}-M\right\|_{s}+$ $\left\|F_{0}-M\right\|_{L^{1}}$ is small, then the initial value problem for $(9.1)$ with the initial condition $F(0, x)=F_{0}(x)$ has a unique global solution $F(t, x)$. For $0 \leqq l \leqq s,\left\|\partial_{x}^{l}(F(t)-M)\right\|_{s-l}$ tends to zero at the rate $t^{-(1 / 2+l) / 2}$ as $t \rightarrow \infty$. This convergence rate is optimal if $\delta_{1}$ and $\delta_{2}$ in $(9.22)$ satisfy $\left(\delta_{1}, \delta_{2}\right) \neq 0$. Moreover, for $0 \leqq l \leqq s-1,\left\|\partial_{x}^{l}(F(t)-\bar{F}(t))\right\|_{s-l}$ tends to zero at the rate $t^{-(1+l) / 2+\alpha}$ as $t \rightarrow \infty$, where $\bar{F}(t, x)$ is the function defined by (9.25) and $\alpha>0$ is a constant determined in Theorem 8.1. Consequently, $\bar{F}(t, x)$ in (9.25) is an asymptotic solution of the one-dimensional Broadwell model (9.1).

\section{Appendix. Reduction to the One-Dimensional Equation}

The general form of the discrete Boltzmann equation in $\mathbb{R}^{n}(n \geqq 2)$ is given by (see $[8,3])$

$$
\frac{\partial N_{p}}{\partial t}+\mathbb{U}_{p} \cdot \nabla N_{p}=\sum_{q, r, s=1}^{J}\left(B_{r s}^{p q} N_{r} N_{s}-B_{p q}^{r s} N_{p} N_{q}\right), \quad p=1, \ldots, J,
$$

where $N_{p}=N_{p}(t, \mathbb{X})$ represents the mass density of gas particles with the velocity $\mathbb{U}_{p}$ (constant vector in $\mathbb{R}^{n}$ ) at time $t \geqq 0$ and position $\mathbb{X} \in \mathbb{R}^{n}, \nabla$ denotes the gradient with respect to $\mathbb{X}$, and the coefficients $B_{r s}^{p q}, p, q, r, s=1, \ldots, J$, are nonnegative constants satisfying

$$
B_{s r}^{p q}=B_{r s}^{p q}=B_{r s}^{q p}, \quad B_{r s}^{p q}=B_{p q}^{r s}
$$

for any $p, q, r, s=1, \ldots, J$. 
We shall explain how the one-dimensional equation (1.1) is derived form (A.1). Let $x \in \mathbb{R}$ be the first component of $\mathbb{X} \in \mathbb{R}^{n}$. We assume that

$$
\begin{gathered}
N_{p}(t, \mathbb{X}), p=1, \ldots, J, \text { are functions depending only on } \\
t \text { and } x \text {, i.e., } N_{p}(t, \mathbb{X})=N_{p}(t, x) .
\end{gathered}
$$

To state the second assumption, we introduce a partition of the set $\{1, \ldots, J\}$. Let $p, q \in\{1, \ldots, J\}$. When the first component of two velocities $\mathbb{U}_{p}$ and $\mathbb{U}_{q}$ are equal and in addition $\left|\mathbb{U}_{p}\right|=\left|\mathbb{U}_{q}\right|$, we write $p \sim q$. It is easily seen that this is an equivalence relation on $\{1, \ldots, J\}$. Hence we have a partition of $\{1, \ldots, J\}$, namely, $\{1, \ldots, J\}=$ $I_{1} \cup \cdots \cup I_{m}$, where $I_{i}, i=1, \ldots, m$, denote the equivalence classes. We then assume that for each $i=1, \ldots, m$,

$$
N_{p}(t, x)=N_{q}(t, x) \text { for any } p, q \in I_{i} .
$$

Under the assumptions (A.3) ${ }_{1,2}$ Eq. (A.1) is reduced to the one-dimensional Eq. (1.1). To see this, we put for each $i=1, \ldots, m$,

$$
\begin{aligned}
F_{i}(t, x) & =N_{p}(t, x) \quad p \in I_{i}, \\
v_{i} & =\text { the first component of } \mathbb{U}_{p}, \quad p \in I_{i}, \\
\alpha_{i} & =\text { the cardinality of } I_{\imath} .
\end{aligned}
$$

Also, we put for each $i, j, k, l=1, \ldots, m$,

$$
A_{k l}^{i j}=\sum B_{r s}^{p q}
$$

where the summation is taken over all $p \in I_{i}, q \in I_{j}, r \in I_{k}$ and $s \in I_{l}$. We sum up the equations in (A.1) for $p \in I_{i}$. Then by the assumptions (A.3) $)_{1,2}$ and the definitions (A.4) and (A.5) ${ }_{1-3}$, we easily obtain (1.1). The condition (1.2) follows from (A.2) and (A.5) . Here we remark that all the $v_{i}, i=1, \ldots, m$, in $(\mathrm{A} .5)_{1}$ are not distinct in general by the definition of the partition of $\{1, \ldots, J\}$.

Finally, we give a remark on summational invariants. A summational invariant of (A.1) is defined as a vector $\Psi={ }^{t}\left(\Psi_{1}, \ldots, \Psi_{J}\right) \in \mathbb{R}^{J}$ satisfying

$$
B_{r s}^{p q}\left(\Psi_{p}+\Psi_{q}-\Psi_{r}-\Psi_{s}\right)=0
$$

for any $p, q, r, s=1, \ldots, J$ (see $[8,3])$. Let $\Psi={ }^{t}\left(\Psi_{1}, \ldots, \Psi_{J}\right)$ be a summational invariant of (A.1) and satisfy $(\mathrm{A} .3)_{2}$, namely, $\Psi_{p}=\Psi_{q}$ for any $p, q \in I_{i}$. Put for each $i=1, \ldots, m$,

$$
\bar{\psi}_{i}=\Psi_{p}, \quad p \in I_{i} .
$$

Summing up (A.6) for $p \in I_{i}, q \in I_{j}, r \in I_{k}$ and $s \in I_{l}$, we get $A_{k l}^{i j}\left(\bar{\psi}_{l}+\bar{\psi}_{j}-\bar{\psi}_{k}-\bar{\psi}_{l}\right)=0$ by (A.5) ${ }_{3}$ and (A.7). Therefore, taking the definition (2.1) into account, we know that $\psi={ }^{t}\left(\alpha_{1} \bar{\psi}_{1}, \ldots, \alpha_{m} \bar{\psi}_{m}\right)$ is a summational invariant of (1.1). Physically reasonable models in $\mathbb{R}^{n}$ usually have the following $n+2$ vectors as summational invariants,

$$
\begin{aligned}
\Psi^{(1)} & ={ }^{t}(1, \ldots, 1), \quad \Psi^{(k+1)}={ }^{t}\left(u_{1 k}, \ldots, u_{J k}\right), \quad k=1, \ldots, n, \\
\Psi^{(n+2)} & ={ }^{t}\left(\left|\mathbb{U}_{1}\right|^{2}, \ldots,\left|\mathbb{U}_{J}\right|^{2}\right),
\end{aligned}
$$

where $u_{p k}$ denotes the $k$-th component of $U_{p}$. Among these summational invariants, 
only $\Psi^{(1)}, \Psi^{(2)}$ and $\Psi^{(n+2)}$ satisfy (A.7) in general by the definition of the partition of $\{1, \ldots, J\}$.

\section{References}

1. Beale, J. T.: Large-time behavior of the Broadwell model of a discrete velocity gas. Commun. Math. Phys. 102, 217-235 (1985)

2. Broadwell, J. E.: Shock structure in a simple discrete velocity gas. Phys. Fluids 7, 1243-1247 (1964)

3. Cabannes, H.: The discrete Boltzmann equation (Theory and applications). Lecture Notes, University of California, Berkeley 1980

4. Cabannes, H.: Comportement asymptotique des solutions de l'équation de Boltzmann discrète. C. R. Acad. Sci. Paris 302, 249-253 (1986)

5. Caflisch, R. E.: Navier-Stokes and Boltzmann shock profiles for a model of gas dynamics. Commun. Pure Appl. Math. 32, 521-554 (1979)

6. Caflisch, R. E., Papanicolaou, G. C.: The fluid-dynamical limit of a nonlinear model Boltzmann equation. Commun. Pure Appl. Math. 32, 589-616 (1979)

7. Ellis, R. S., Pinsky, M. A.: Limit theorems for model Boltzmann equations with several conserved quantities. Indiana Univ. Math. J. 23, 287-307 (1973)

8. Gatignol, R.: Théorie cinétique des gaz à répartition discrète de vitesses. Lecture Notes in Physics, Vol. 36. Berlin, Heidelberg, New York: Springer 1975

9. Hamdache, K.: Existence globale et comportement asymptotique pour l'équation de Boltzmann à répartition discrète des vitesses. J. Mécan. Thèor Appl. 3, 761-785 (1984)

10. Inoue, K., Nishida, T.: On the Broadwell model of the Boltzmann equation for a simple discrete velocity gas. Appl. Math. Opt. 3, 27-49 (1976)

11. Kato, T.: Perturbation theory for linear operators. (Sec. ed.) Berlin, Heidelberg, New York: Springer 1976

12. Kawashima, S.: The asymptotic equivalence of the Broadwell model equation and its Navier-Stokes model equation. Jpn. J. Math. 7, 1-43 (1981)

13. Kawashima, S.: Global existence and stability of solutions for discrete velocity models of the Boltzmann equation, Recent topics in nonlinear PDE, Lecture Notes in Num. Appl. Anal 6, Kinokuniya, 1983 , pp. 59-85

14. Kawashima, S.: Large-time behavior of solutions for hyperbolic-parabolic systems of conservation laws and applications. Proc. R. Soc. Edinburgh. (to appear)

15. Lax, P. D.: Hyperbolic systems of conservation laws, II. Commun. Pure Appl. Math. 10, 537-566 (1957)

16. Liu, T.-P.: Nonlinear stability of shock waves for viscous conservation laws, Memoirs. Am. Math. Soc., No. 328, Vol. 56 (1985)

17. Shizuta, Y., Kawashima, S.: Systems of equations of hyperbolic-parabolic type with applications to the discrete Boltzmann equation. Hokkaido Math. J. 14, 249-275 (1985)

18. Tartar, L.: Some existence theorems for semilinear hyperbolic systems in one space variables, MRC Technical Summary Report, University of Wisconsin 1980

19. Umeda, Y., Kawashima, S., Shizuta, Y.: On the decay of solutions to the linearized equations of electro-magnetofluid dynamics. Jpn. J. Appl. Math. 1, 435-457 (1984)

Communicated by C. H. Taubes

Received July 15, 1986 
